JGR Planets

\author{
RESEARCH ARTICLE \\ 10.1029/2019JE005932 \\ Key Points: \\ - We used the MDIS-WAC data to \\ produce an eight-color mosaic of the \\ Shakespeare quadrangle \\ - We identified spectral units from the \\ maps of Shakespeare \\ - We selected two regions of high \\ interest as potential targets for the \\ BepiColombo mission
}

Correspondence to:

N. Bott,

nicolas.Bott@obspm.fr

Citation:

Bott, N., Doressoundiram, A., Zambon, F., Carli, C., Guzzetta, L., Perna, D., \& Capaccioni, F. (2019). Global spectral properties and lithology of Mercury: The example of the Shakespeare (H-03) quadrangle. Journal of Geophysical Research: Planets Planets, 124, 2326-2346. https://doi.org/10.1029/2019JE005932

Received 28 JAN 2019 Accepted 25 JUL 2019 Accepted article online 1 AUG 2019 Published online 2 SEP 2019

\section{Global Spectral Properties and Lithology of Mercury: The Example of the Shakespeare (H-03) Quadrangle}

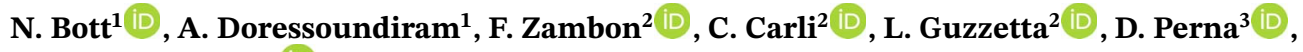 \\ and F. Capaccioni ${ }^{2}$ iD \\ ${ }^{1}$ LESIA-Observatoire de Paris-CNRS-Sorbonne Université-Université Paris-Diderot, Meudon, France, ${ }^{2}$ Istituto di \\ Astrofisica e Planetologia Spaziali-INAF, Rome, Italy, ${ }^{3}$ Osservatorio Astronomico di Roma-INAF, Monte Porzio \\ Catone, Italy
}

\begin{abstract}
The MErcury Surface, Space ENvironment, GEochemistry and Ranging mission showed the surface of Mercury with an accuracy never reached before. The morphological and spectral analyses performed thanks to the data collected between 2008 and 2015 revealed that the Mercurian surface differs from the surface of the Moon, although they look visually very similar. The surface of Mercury is characterized by a high morphological and spectral variability, suggesting that its stratigraphy is also heterogeneous. Here, we focused on the Shakespeare (H-03) quadrangle, which is located in the northern hemisphere of Mercury. We produced an 8-color cube of this quadrangle at 450-m per pixel spatial resolution and with a complete coverage. Various selected color maps based on this eight-color cube were used to analyze the spectral properties of this region to define its compositional variability and identify some clear units constrained by relevant spectral parameters: for example, we identified a higher concentration of low reflectance material around three main craters of Shakespeare (Degas, Akutagawa, and Sholem Aleichem) and in the area to the south of Sobkou Planitia delimited by $24.4^{\circ} \mathrm{N}$ to $28.4^{\circ} \mathrm{N}$ and $-140^{\circ} \mathrm{W}$ to $-125^{\circ} \mathrm{W}$. Moreover, we selected some regions of interest, which can be proposed as particularly interesting targets for the Visual and Infrared Hyper-spectral Imager and Mercury Radiometer and Thermal Imaging Spectrometer instruments onboard the BepiColombo spacecraft. This work can help for the geological analysis of this quadrangle, by integrating information that are usually not derived with a single morphostratigraphic analysis.
\end{abstract}

Plain Language Summary The MErcury Surface, Space ENvironment, GEochemistry and Ranging mission showed Mercury's surface with an accuracy never reached before. The analyses performed thanks to the data collected between 2008 and 2015 revealed that the Mercurian surface differs from the surface of the Moon, although they look visually very similar. Mercury's surface is characterized by a high variability in terms of shape and colors, suggesting that its subsurface is also heterogeneous. Here, we focused on the region called Shakespeare, which is located in the north hemisphere of Mercury. We produced a mosaic of images of this region for several colors at high resolution and with a complete coverage. Various selected color maps based on this mosaic were used to analyze the properties of this area to define its compositional variability: for example, we identified a higher concentration of dark material around three craters of Shakespeare (Degas, Akutagawa, and Sholem Aleichem) and in the south of the Sobkou basin. Moreover, we selected some interesting regions that can be proposed as particularly interesting targets for instruments onboard the BepiColombo spacecraft. This work can help for the geological analysis of this quadrangle, by adding information that are usually not derived with a single analysis of landforms.

\section{Introduction}

One of the goals of the MErcury Surface, Space ENvironment, GEochemistry and Ranging (MESSENGER) mission (Solomon et al., 2007) was to better understand its internal and surface composition. The very low abundance in iron (ions and metallic) of the surface has been confirmed (McClintock et al., 2008; Weider et al., 2012, 2014), and the volatile elements in high quantities (Nittler et al., 2011; Peplowski et al., 2011; Weider et al., 2012, 2015) compared to the one of the Moon (Lucey et al., 1995) were unexpected. The Mercury Dual Imaging System (MDIS) instrument, thanks to its accurate (see Table 1) Wide-Angle Camera
(C)2019. American Geophysical Union. All Rights Reserved. 


\begin{tabular}{|c|c|c|}
\hline \multicolumn{3}{|c|}{$\begin{array}{l}\text { Table } 1 \\
\text { Comparison of the Spatial Resolution, Number of Colors, and Cover } \\
\text { Television Science and the MESSENGER MDIS-WAC Instruments }\end{array}$} \\
\hline \multirow{3}{*}{ Instrumental parameter } & Mariner 10 & \\
\hline & Television Science & MESSENGER \\
\hline & (Davies et al., 1978) & MDIS-WAC ${ }^{\mathrm{a}}$ \\
\hline \multirow[t]{2}{*}{ Spatial resolution } & $100 \mathrm{~m}$ to $10 \mathrm{~km}$ per pixel & $8 \mathrm{~m}$ to $7 \mathrm{~km}$ per pixel \\
\hline & 3 & 11 \\
\hline \multirow[t]{3}{*}{ Number of colors } & $(355,475$, and $575 \mathrm{~nm})$ & $(433.2,479.9,558.9,628.8$ \\
\hline & & 698.8, 748.7, 828.4, 898.8, \\
\hline & & $947.0,996.2$, and $1,012.6 \mathrm{~nm})$ \\
\hline Coverage & $45 \%$ & $100 \%$ \\
\hline
\end{tabular}

Note. The multifilters camera of MESSENGER provided a better spatial resolution, number of colors and fully mapped the surface of Mercury. MESSENGER = MErcury Surface, Space ENvironment, GEochemistry and Ranging.

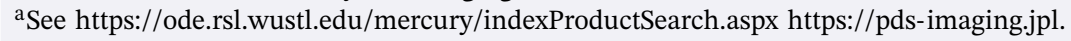
nasa.gov/search/, and https://pdsimage2.wr.usgs.gov/data/mess-h-mdis-5-rdr-bdr-v1.0/ MSGRMDS_4001/EXTRAS/ websites.

(WAC) and Narrow-Angle Camera, revealed a very dark surface (Domingue et al., 2011, retrieved a bolometric albedo of 0.081 over the wavelength range from 430 to $1010 \mathrm{~nm}$ ), with high geological and spectral variabilities. Spudis and Guest (1988) and Trask and Guest (1975) defined three main geological units based on the morphology and the texture of the terrains: the young smooth plains (SP), the intermediate plains (ImP), and the oldest intercrater plains (IcP). Besides, based on the spectral slope and the relative reflectance, Denevi et al. (2009) identified three terrains that can be attributed to SP: the high-reflectance red plains (HRP), the intermediate terrains (IT) and the low-reflectance blue plains (LBP). Furthermore, (Blewett et al., 2009; Robinson et al., 2008) pointed out several minor color units: three types of red spots (RS), among which pyroclastic deposits (PD), which have the reddest spectral slopes on Mercury, and bright crater floor deposits (BCFD), composed of hollows which have bluer slopes (Blewett et al., 2011, 2013, 2016).

The space weathering of ferrous iron in minerals produces notably nanophase metallic iron, which darkens the surface of Mercury (Blewett et al., 2009). However, this point associated to the low amount of iron on Mercury can not fully explain the low albedo of Mercury. This led to the need to find a darkening agent in addition to iron. A good candidate can be found in the low reflectance material (LRM) identified thanks to the MESSENGER mission. The LRM has been investigated through MDIS (Klima et al., 2018; Murchie et al., 2015; Thomas et al., 2016; Xiao et al., 2013) data, Mercury Atmosperic and Surface Composition Spectrometer data (Izenberg et al., 2014; Thomas et al., 2016; Trang et al., 2017), and Gamma Ray and Neutron Spectrometer data (Peplowski et al., 2016). Thus, particular spectral properties of this material have been raised: It consists of a dark (hence low reflectance) material, with one of the shallowest spectral slopes of the Mercurian surface, which samples carbon-bearing deposits within the crust during impacts (Peplowski et al., 2016; Vander Kaaden \& McCubbin, 2015). This led to the hypothesis that carbon could be the darkening agent. However, Blewett et al. (2013) suggested that microsized and nanosized particules of sulfides (e.g., FeS and CaS) can also be involved in the darkening agent responsible for the low reflectance of LRM terrains. The concentration of hollows on LRM terrains (Blewett et al., 2011, 2013; Thomas et al., 2016) and the first detection ever in hollows of a weak mineral absorption band at $600 \mathrm{~nm}$ attributed to sulfides (Vilas et al., 2016) mixed with bedrock-forming minerals like pyroxenes including elements such as chromium, titanium, and nickel (Lucchetti et al., 2018) strengthened this hypothesis. Moreover, the sublimation of the volatile sulfides would be related to the formation of hollows (Blewett et al., 2013). Furthermore, the ejecta diversity, bright in some areas $(\approx 40 \%$ brighter than the average terrains at $749 \mathrm{~nm})$, dark in other regions $(\approx 20 \%$ darker at $749 \mathrm{~nm}$ ), is also indicative of a very complex Mercurian crust. Hence, a more closer investigation of the lithology of the surface is required.

In this work, we focus on the Shakespeare (H-03) quadrangle. We compared the terrain units identified mainly on a morphological basis (see Guzzetta et al., 2017, and Figure 1) and the respective spectral responses, in order to constrain the mapped units also from a compositional point of view and then 


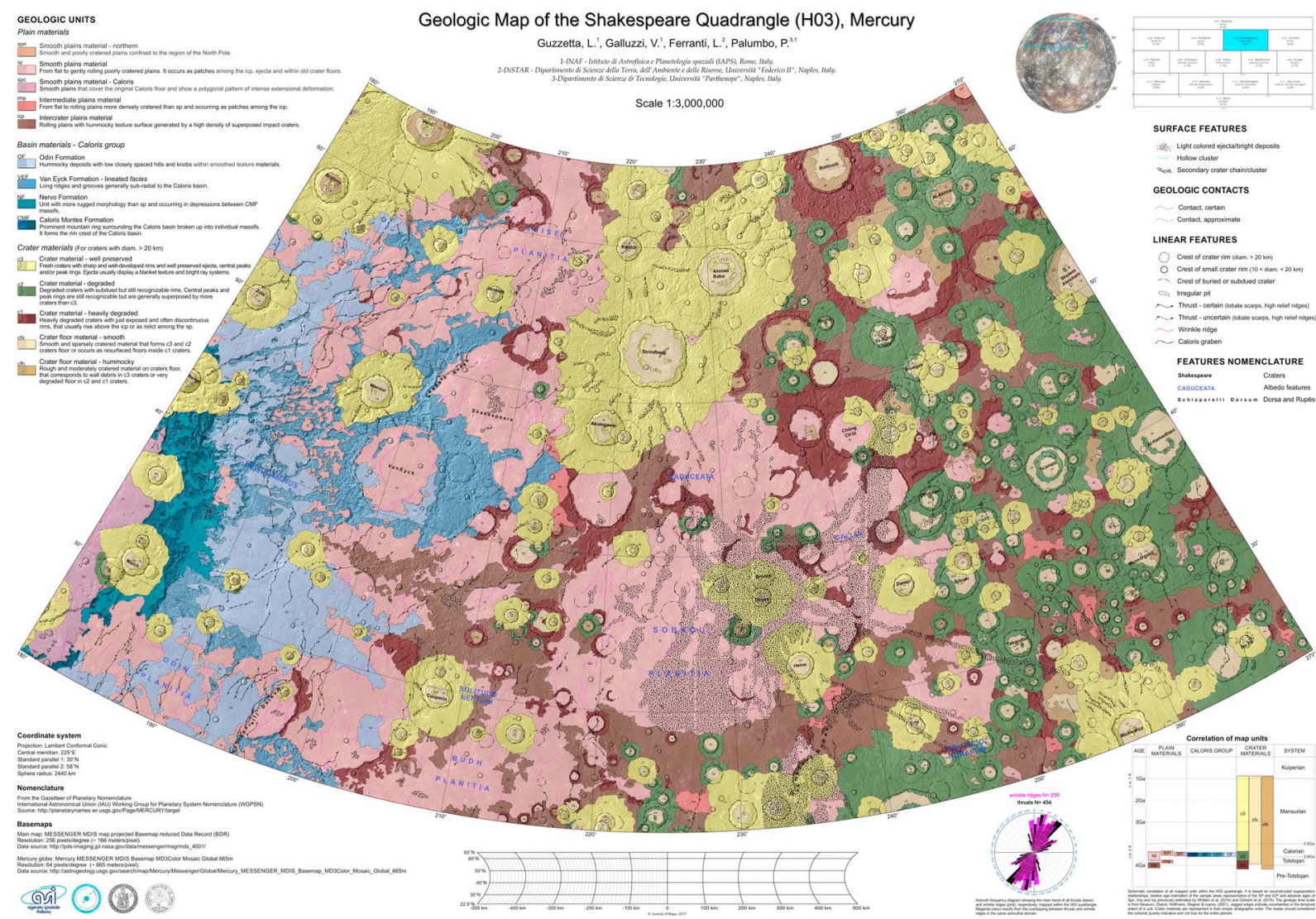

Figure 1. The geological map of the Shakespeare quadrangle published in Guzzetta et al. (2017).

geological units, and help for a more accurate geological analysis of this quadrangle, integrating information that are usually not derived with a single morphostratigraphic analysis.

An eight-color mosaic of the Shakespeare quadrangle was produced using the Integrated Software for Imagers and Spectrometers software. Then, several spectral analyses were conducted in order to reconstruct the lithology of the studied area. In section 2 are shortly described the data set and the methodology used to obtain our eight-color mosaic of Shakespeare. Section 3 deals with the spectral analysis of our product, and the results are exposed in section 4. Finally, in section 5, we discuss the results of the spectral analysis, first at a global scale, then drawing our attention on two interesting regions, which can be considered as some targets for the SIMBIO-SYS (Spectrometers and Imagers for Mercury Planetary Orbiter BepiColombo Integrated Observatory SYStem) instrument onboard the BepiColombo spacecraft.

\section{Data Set and Data Processing}

We used the Experiment Data Record obtained by MDIS-WAC (Hawkins et al., 2007) composed of 12 filters, observing Mercury in the visible and near-infrared range, from 433.2 to $1012.6 \mathrm{~nm}$. The characteristics of each filter is detailed in Table 2.

The Shakespeare quadrangle extends from $22.5^{\circ} \mathrm{N}$ to $65^{\circ} \mathrm{N}$ in latitude and from $180^{\circ} \mathrm{E}$ to $270^{\circ} \mathrm{E}$ in longitude and covers an area of about $5 \times 10^{6} \mathrm{~km}^{2}$. The 1:3M-scale geological map of the quadrangle (Guzzetta et al., 2017, and Figure 1) shows the distribution of the terrain units and of the impact craters in this region of the planet. In particular, the main units occurring in Shakespeare are the SP and the IcP, whereas the ImP occur only as small patches (Guzzetta et al., 2017; Spudis \& Guest, 1988; Trask \& Guest, 1975). The impact craters are more abundant in the eastern part of the quadrangle, which thus seems to be older than the western area, dominated by the Caloris ejecta (McCauley et al., 1981).

The final image mosaic product has been obtained in June 2018 with the version 3.5.2.0 (6 April 2018) of the Integrated Software for Imagers and Spectrometers developed and provided by the U.S. Geological Survey 
Table 2

Basic Information on MDIS-WAC Filters (Hawkins et al., 2007)

\begin{tabular}{lccc}
\hline Filter & Wavelength $(\mathrm{nm})$ & Width $(\mathrm{nm})$ & \# images \\
\hline A & 698.8 & 5.3 & 49 \\
B & 700 & 600.0 & 40 \\
C & $\mathbf{4 7 9 . 9}$ & $\mathbf{1 0 . 1}$ & $\mathbf{7 7 2}$ \\
$\mathbf{D}$ & $\mathbf{5 5 8 . 9}$ & $\mathbf{5 . 8}$ & $\mathbf{9 9 6}$ \\
$\mathbf{E}$ & $\mathbf{6 2 8 . 8}$ & $\mathbf{5 . 5}$ & $\mathbf{7 7 0}$ \\
$\mathbf{F}$ & $\mathbf{4 3 3 . 2}$ & $\mathbf{1 8 . 1}$ & $\mathbf{2 3 0 0}$ \\
$\mathbf{G}$ & $\mathbf{7 4 8 . 7}$ & $\mathbf{5 . 1}$ & $\mathbf{6 1 6 3}$ \\
H & 947.0 & 6.2 & 48 \\
I & $\mathbf{9 9 6 . 2}$ & $\mathbf{1 4 . 3}$ & $\mathbf{2 3 0 5}$ \\
J & $\mathbf{8 9 8 . 8}$ & $\mathbf{5 . 1}$ & $\mathbf{7 5 9}$ \\
K & 1012.6 & 33.3 & 48 \\
L & $\mathbf{8 2 8 . 4}$ & $\mathbf{5 . 2}$ & $\mathbf{9 9 2}$ \\
\hline Note. The filters used to build the mosaic are marked in bold. Last column \\
is the number of images available for the Shakespeare quadrangle. MDIS \\
= Mercury Dual Imaging System; WAC = Wide-Angle Camera.
\end{tabular}

(Anderson et al., 2004; Gaddis et al., 1997; Torson \& Becker, 1997). Our method is mainly based on the method described in Denevi et al. (2018, section 8) to obtain the final products of the MESSENGER team. Below are reminded the main steps of the two methods, and their differences are addressed.

The Planetary Data System (available in https://pds-imaging.jpl.nasa.gov/Atlas/) images were first georeferenced using SPICE kernels integrated to the software and a shape model of Mercury. This geometric calibration uses notably the last version of Planetary Constants Kernel as suggested in the comments on the MESSENGER SPICE data archive. The shape model is a digital elevation model produced at DLR (Preusker et al., 2017). This digital elevation model has a high vertical accuracy $(30 \mathrm{~m})$ and a better spatial resolution (222 m per pixel) than the U.S. Geological Survey shape model (665 m/pixel spatial resolution). The distance to the center of the planet varies from $2,435,706$ to $2,442,822 \mathrm{~m}$, that is, an elevation $\left(R_{\text {Mercury }}=2,439,400 \mathrm{~m}\right)$ ranging from $-3,694$ to $+3,422 \mathrm{~m}$.

Then a radiometric calibration was applied, including the correction of the dark current, of the linearity, of the smear, and of the flat field of the images. The use of the appropriate command called "mdiscal" performs the same calibration routine than in the Calibrated Data Record catalog file available on the Orbital Data Explorer. This includes in particular the empirical corrections for responsivity issues.

We selected only 8 filters among the 12 available for MDIS-WAC. Filters A, B, H, and K (698.8, 700.0, 947.0, and $1012.6 \mathrm{~nm}$ ) do not ensure a complete coverage of the quadrangle. Moreover, filter B (700 nm) was used for the calibration operations. The images with too high illumination conditions (incidence angle $i>70^{\circ}$, emission angle $e>70^{\circ}$ ) were discarded to avoid a too big difference between the illumination conditions of two overlapped images. Without this filtering, the photometric correction is not fully efficient, that is, some image footprints are visible in the final mosaic. Finally, we reduced the amount of data to improve the processing time, as follows: first, we sorted our images by increasing spatial resolution. Then we considered the footprint of each image, one by one: If the footprint of an image overlaps more than $90 \%$ of the already present footprints, then this image was skipped. We considered $90 \%$ as a relevant threshold, because with a smaller value, some holes appeared in the final product and with a higher one, too many images were kept, and so the filtering was not efficient. With this filtering, the number of cubes used for the mosaic has been reduced from 759 to 499 cubes, thus dividing by $\sim 1.5$ the total processing time.

We applied a Kaasalainen-Shkuratov model (Kaasalainen et al., 2001; Shkuratov \& Helfenstein, 2001) to normalize our images to the geometric conditions commonly used in laboratory experiments, that is, incidence angle $i=30^{\circ}$, emission angle $e=0^{\circ}$, and phase angle $\alpha=30^{\circ}$. This model gives a better global photometric correction, in particular, for seams between images of different illumination conditions (Domingue et al., 2016), than the Hapke model (Domingue et al., 2015; Hapke, 1981; Hapke et al., 2012). 
Two different projections were considered: an equirectangular projection to keep possible a comparison with the eight-color mosaic produced by the MESSENGER team and a Lambert conformal conic projection, which is the most conventional projection at these latitudes. The resolution of each image has been forced to $450 \mathrm{~m}$ per pixel. This spatial resolution has been chosen for two reasons: first, to be consistent with the resolution selected by (Zambon et al., 2017) for their own eight-color mosaics (Victoria and Hokusai quadrangles); then, because it is close to the average spatial resolution of the images used to produce our mosaic. The projected images were then coregistered.

Finally, we produced the eight-color mosaic and we filtered it to remove the bad pixels still present (e.g., negative pixel values or greater than 1), which mainly come from the photometric correction. We did not consider the scattered light issue as in Denevi et al. (2018) because of the small redundancy of our data set. However, the problem should be reduced thanks to the final bad pixels filtering.

\section{Spectral Analysis}

To have a global view of the spectral and lithological properties of the surface of Mercury at a quadrangle scale, we performed thresholds of different parameters highlighting variations in reflectance, spectral slope, ratios, and spectral curvature. Moreover, we used red-green-blue (RGB) combinations to emphasize spectral variations and we took into account the information obtained by a principal component analysis (PCA). PCA is a statistical method that converts a data set of potentially correlated variables into a data set of uncorrelated variables called principal components (PC). This method produces the least number of relevant parameters to study the global spectral properties of the data set. It has been used several times to identify specific terrains on Mercury (Denevi et al., 2009; Peplowski \& Stockstill-Cahill, 2018; Robinson et al., 2008).

To find the PC of a data set, the basic method using PCA can be based on either a covariance matrix or a correlation matrix. The correlation matrix is used if the variances of the individual variables differ significantly or if the variables have different units of measurement. Since the variables of our data set are of the same physical quantity (reflectance) and have the same unit of measurement (dimensionless physical quantity), we used the PCA which computes the covariance matrix for our analyses. In our context, applied to our 8-color mosaic, the first PC (PC1) gives information on the reflectance variations, whereas the second $\mathrm{PC}$ (PC2) shows spectral variations related to the physical state or chemistry of the material, that is, predominant slope of the spectral continuum. We computed the PC of our eight-color mosaic with ENVI .

Combining the information described above from different analyses, we could highlight specific spectral units to be associated to compositional information. In the following subsections, we describe the performed analyses.

\subsection{Classification Using Thresholding}

We retained four spectral parameters for this analysis: PC2, reflectance at $749 \mathrm{~nm}$, ratio 996/433 nm and spectral curvature around $600 \mathrm{~nm}$. Here we show the thresholding of the two first parameters, which are the best to highlight the two main spectral properties of the Mercurian surface (for further details, see Murchie et al., 2015, who used these parameters for their investigation of the color units of Mercury): the thresholding of the reflectance at $749 \mathrm{~nm}$ emphasizes the brightness variations of the Mercurian surface, while the thresholding of PC2 puts forward its global spectral slope variations. The two other spectral parameters were considered to complete both the global information given by the thresholdings and the discussion. This way, we aimed to identify units associated to specific terrains with probable differences in composition.

For each spectral parameter, we started considering the lowest significant values of this parameter, that is, the values from which a specific terrain starts to appear. Then, we increased progressively the value of the parameter till another well separated terrain clearly emerges. The value corresponding to that change of terrain is taken as the upper limit of the threshold. We proceeded this way till the highest significant values of the spectral parameter are reached. The thresholding method enables to identify some terrains at large scale, although it is not possible to discriminate specific terrains when considering values of the parameter close to the mean value. The terrains which cannot be separated are gathered in an unique, widespread region labeled as "Other," and can be considered as an intermediate region.

The ratio $996 / 433 \mathrm{~nm}$ is another manner to highlight the spectral slope variations. The ratio $R$ of two bands $R_{1}$ at $\lambda_{1} \mathrm{~nm}$ and $R_{2}$ at $\lambda_{2} \mathrm{~nm}$ of the eight-color mosaic is linked to the spectral slope $S$ by

$$
R=\frac{R_{1}}{R_{2}}=1+\frac{\lambda_{2}-\lambda_{1}}{R_{1}} S
$$



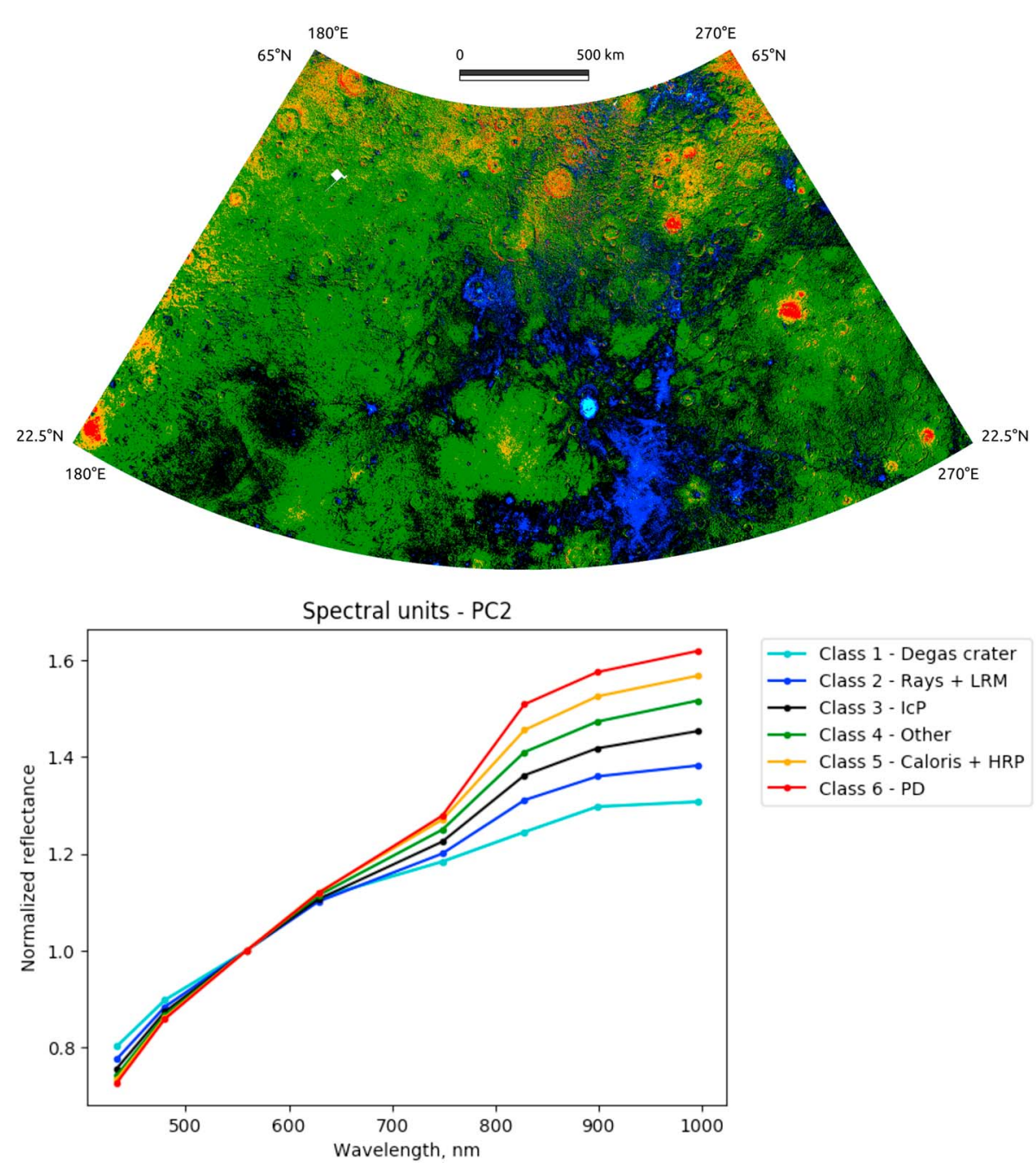

Figure 2. Top: The spectral map of the Shakespeare quadrangle for the PC2. Bottom: Average spectra (normalized at $559 \mathrm{~nm}$ ) of each spectral unit of the previous map. LRM = low reflectance material; IcP = intercrater plains; HRP = high-reflectance red plains; $\mathrm{PD}=$ pyroclastic deposits.

It is important to notice here that the relationship between the ratio and the spectral slope is not linear since the reflectance $\left(R_{1}\right)$ is involved. For the same spectral slope, a brighter terrain will have a lower ratio. Ratios are thus also useful to enhance the bluest and brightest, so freshest material like ejecta and rays, what was not possible with the spectral slope alone.

Vilas et al. (2016) and Lucchetti et al. (2018) showed that a weak, broadband around $600 \mathrm{~nm}$ can be detected on the floor of some craters containing hollows, which contain fresh material according to (Blewett et al., 2011). This band is expected to be associated to a material present in the darkest regions of Mercury, that is, those that may contain LRM. Klima et al. (2018) used the spectral curvature this band creates to detect LRM on the surface of Mercury. To do a finer analysis of this spectral curvature, we considered a spectral parameter introduced by Murchie et al. (2015) and used recently by Klima et al. (2018) to compute the spectral curvature at $600 \mathrm{~nm}$ (equation (2)).

$$
S C_{600}=1-\frac{\left(R_{559}+R_{629}+R_{749}+R_{828}\right) / 4}{\left(R_{899}+R_{479}\right) / 2}
$$

\subsection{RGB Mapping}

We combined several spectral parameters in one map by doing a RGB color mapping to find the right color combinations to enhance the composition variations across the surface. This way, it is easier to identify regions of interest and do a finer analysis or classification. 

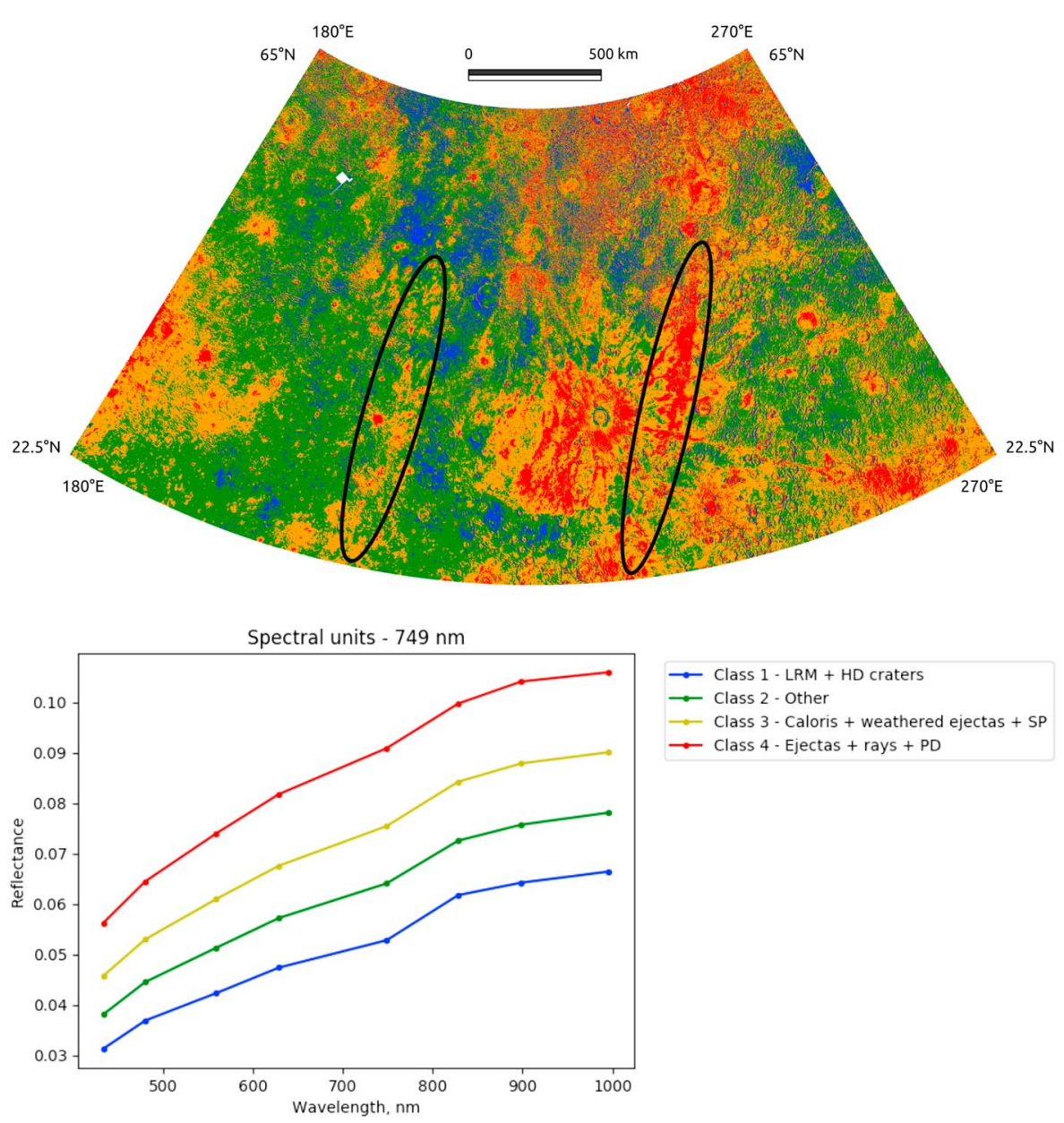

$\rightarrow$

$\rightarrow$ Class 4 - Ejectas + rays + PD

Figure 3. Top: The spectral map of the Shakespeare quadrangle for the reflectance at $749 \mathrm{~nm}$. Bottom: Average spectra of each spectral unit of the previous map. LRM = low reflectance material; HD craters = heavily degraded craters; $\mathrm{SP}=$ smooth plains; $\mathrm{PD}=$ pyroclastic deposits.

Two RGB maps were commonly used in the literature (Blewett et al., 2009; Denevi et al., 2009; Robinson et al., 2008): The first combines the reflectance at 996,749 , and $433 \mathrm{~nm}$, respectively; the second includes the two first principal components (PC1 and PC2) and the ratio 433/996 nm. On the first map, only reflectance can be directly visualized. Yet, as evidenced in section 3.1, the spectral slope is also essential to better constrain the lithology of a surface. To have an access to the spectral slope distribution across the quadrangle, it is useful to do the ratio between two filters (e.g., 996/433 nm).

We also looked for the potential spectral signature of the surface of Mercury in the visible domain, that is, the spectral curvature at $600 \mathrm{~nm}$. To do so, we considered the parameter $S C_{600}$ (defined in section 3.1) to search for areas where this curvature is significant. As employed by Pieters et al. (1994) for their RGB map to estimate the abundance of iron on the Moon, we decided to introduce the reciprocal of $S C_{600}$, to be more sensible to the weak strength of this curvature.

Finally, we defined an RGB map similar to the one using PC2 and PC1 for the red and green channels, respectively, but the spectral curvature at $600 \mathrm{~nm}$ for the blue channel.

\subsection{K-Mean Clustering}

We also performed a K-mean clustering on selected areas of the quadrangle, whose aim is to classify a variable into $\mathrm{k}$ clusters. This classification method has been introduced by Tou and Gonzalez (1974). Here the variable is the multicolor spectrum coming from a pixel of our eight-color mosaic. In addition, we used an algorithm (Marzo et al., 2006, 2008, 2009) based on the calculation of the Calinski-Harabasz criterion (shortened CH criterion hereafter; Calinski \& Harabasz, 1974) to determine if there is a clustering structure. If yes, it will give the natural number of clusters for the data set considered, making the process fully unsupervised. 
Table 3

Thresholds of PC2 Used to Classify Shakespeare as Visible in Figure 2

\begin{tabular}{lcccc}
\hline & & \multicolumn{2}{c}{ Spectral parameter range } & \\
\cline { 2 - 4 } Threshold \# & Color on map & Min & Max & Terrain/feature associated \\
\hline 1 & Cyan & -0.022 & -0.01 & Degas crater \\
2 & Blue & -0.01 & -0.005 & Rays, LRM \\
3 & Black & -0.005 & -0.001 & IcP \\
4 & Green & -0.001 & 0.0035 & Other \\
5 & Orange & 0.0035 & 0.007 & Caloris, HRP \\
6 & Red & 0.007 & 0.018 & Pyroclastic deposits \\
\hline
\end{tabular}

Note. $\mathrm{LRM}=$ low reflectance material; HRP $=$ high-reflectance red plains; $\mathrm{IcP}=$ intercrater plains; $\mathrm{PC}=$ principal component.

A data set has a clustering structure if the $\mathrm{CH}$ criterion shows a local maximum. Thus, the region of interest is separated into clusters. Each cluster is characterized by an average spectrum, revealing the spectral variability of the region of interest.

This method is different from the PCA. Indeed, where PCA is a method of data reduction, so better used at large scale to remove the less significant points of the data set, the K-mean clustering will only separate the data set into several groups, making it more usable for data set with limited size.

\section{Results}

\subsection{Using the Thresholding}

Figures 2 and 3 show the maps obtained by thresholding PC2 and the reflectance at $749 \mathrm{~nm}$, respectively. Tables 3 and 4 detail these thresholdings. The mosaics of the four first PCs (PC1, PC2, PC3, and PC4) of the Shakespeare quadrangle are shown in Appendix A.

The thresholding permits to identify geological terrains mapped by Guzzetta et al. (2017). Intercrater plains, identified as Class 3 in Figure 2, are the most convincing example. Indeed, the terrains surrounding Sobkou Planitia which have been mapped as IcP (see Guzzetta et al., 2017) are very well characterized by a PC2 ranging from -0.005 to -0.001 , according to our map. LRM and the main Hokusai ray coming from the eponymous crater (see section 5.3.2 for more information on this crater) are clearly distinguished through Class 2. For clarity of what follows, since this ray is orthogonal to the Degas rays, we hereafter refer to it as "orthogonal ray," shortened OR. Some patches of Caloris smooth plains material and HRP are detected in the far western of the Shakespeare quadrangle with Class 5. Furthermore, it is also possible to enhance more local features. It appears that the material on the floor and in the close surroundings of Degas crater $\left(37.1^{\circ} \mathrm{N}\right.$, $-127.3^{\circ} \mathrm{W}$ ) is distinct from the rest of the quadrangle, in terms of PC2 values. Hence, we mapped this crater as a separate class. Looking at the average spectrum of class 1 , we can even claim that the material covering the floor of Degas has the flattest spectrum of the Shakespeare quadrangle. Pyroclastic deposits (Besse et al., 2015; Goudge et al., 2014; Head et al., 2008, 2009; Kerber et al., 2009, 2011) are another example of features which constitute a separated class (class 6), but unlike Degas, they have the steepest spectral slope of the Shakepeare region.

Table 4

Thresholds of Reflectance at $749 \mathrm{~nm}$ Used to Classify Shakespeare as Visible in Figure 3

\begin{tabular}{|c|c|c|c|c|}
\hline \multirow[b]{2}{*}{ Threshold \# } & \multirow[b]{2}{*}{ Color on map } & \multicolumn{2}{|c|}{ Spectral parameter range } & \multirow[b]{2}{*}{ Terrain/feature associated } \\
\hline & & Min & Max & \\
\hline 1 & Blue & 0.035 & 0.058 & LRM, heavily degraded craters \\
\hline 2 & Green & 0.058 & 0.069 & Other \\
\hline 3 & Orange & 0.069 & 0.085 & Caloris, weathered ejecta, SP \\
\hline 4 & Red & 0.085 & 0.102 & ejecta, rays, pyroclastic deposits \\
\hline
\end{tabular}

Note. LRM $=$ low reflectance material. 

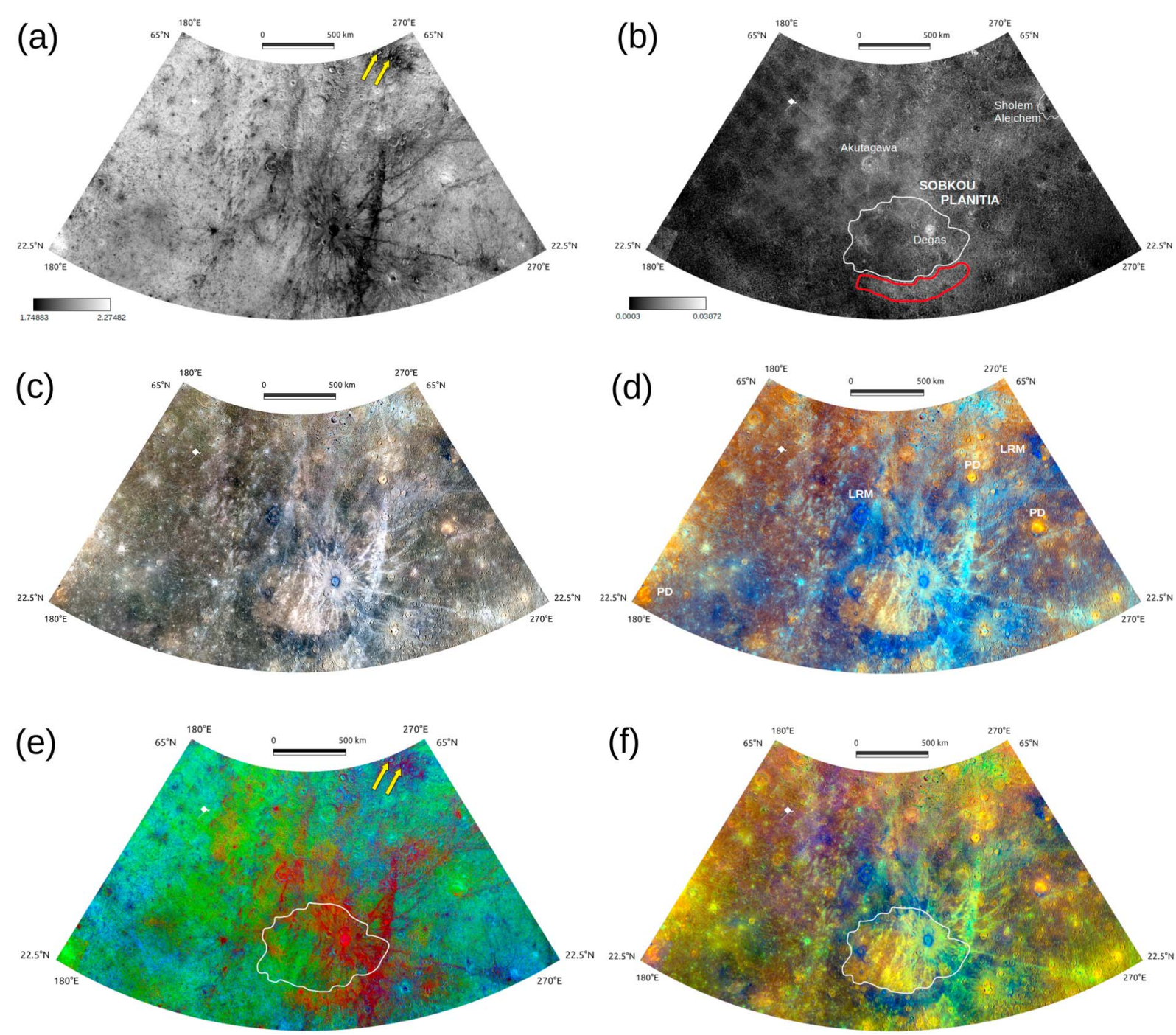

Figure 4. (a) Ratio (996/433 nm) map of the Shakespeare quadrangle. The yellow arrows point the two unnamed Kuiperian craters discussed in sections 4.2 and 5.2. (b) Map showing the spectral curvature around $600 \mathrm{~nm}$ across the Shakespeare quadrangle. A stretching has been applied to keep values between $0 \%$ and 5\%. (c) RGB $(R=996, G=749$, and $B=433 \mathrm{~nm})$ map of the Shakespeare quadrangle. Red range: 0.06254-0.11021; green range: 0.04946-0.09450; blue range: 0.02909-0.05932. (d) RGB $(\mathrm{R}=\mathrm{PC} 2, \mathrm{G}=\mathrm{PC} 1$, and $\mathrm{B}=433 / 996 \mathrm{~nm})$ map of the Shakespeare quadrangle. Red range: 0.03130-0.05270; green range: 0.13410-0.24909; blue range: 0.43716-0.56937. Annotations: $\mathrm{PD}=$ pyroclastic deposits; $\mathrm{LRM}=$ low reflectance material. $(\mathrm{e}) \mathrm{RGB}\left(\mathrm{R}=S C_{600}, \mathrm{G}=996 / 433 \mathrm{~nm}\right.$, and $\left.\mathrm{B}=1 / S C_{600}\right)$ map of the Shakespeare quadrangle. Red range: 0.0003-0.03872; green range: 1.74883-2.27482; blue range: 20.03125-13331. The yellow arrows point the two unnamed Kuiperian craters discussed in sections 4.2 and 5.2. (f) $R G B\left(R=P C 2, G=P C 1\right.$, and $\left.B=S C_{600}\right)$ map of the Shakespeare quadrangle. Red range: 0.03134-0.05273; green range: 0.13435-0.24914; blue range: 0.0003-0.03872. RGB = red-green-blue.

Other terrains can be identified with the thresholding of the reflectance at $749 \mathrm{~nm}$, like the heavily degraded craters material, what we could not do with PC2. Those terrains are quite well characterized in the western part of the quadrangle, where no bright features covered them. We also notice that the two main spots of LRM in Shakespeare, close to Akutagawa $\left(50.9^{\circ} \mathrm{N},-90.5^{\circ} \mathrm{W}\right)$ and Sholem Aleichem $\left(48.3^{\circ} \mathrm{N},-141.1^{\circ} \mathrm{W}\right)$ craters, are mapped in class 1 of reflectance (see Figure 3), whereas the LRM which circles the Degas crater is not. Therefore, the LRM identified close to Akutagawa and Sholem Aleichem is brighter than the LRM surrounding Degas. Besides, we can identify the two brightest rays coming from the Hokusai crater in our quadrangle (circled in black in Figure 3; see Ernst et al., 2018; Figure 1 for the complete rays system of this crater): one in the eastern part, which is very bright, so classified in class 4; and one in the western part, less bright and classified in class 3 .

Figure 4a shows the ratio between the reflectance at $996 \mathrm{~nm}$ and the reflectance at $433 \mathrm{~nm}$. As mentioned in section 3.1, a ratio map can be used as a proxy to put forward the global spectral slope variations, that is, 


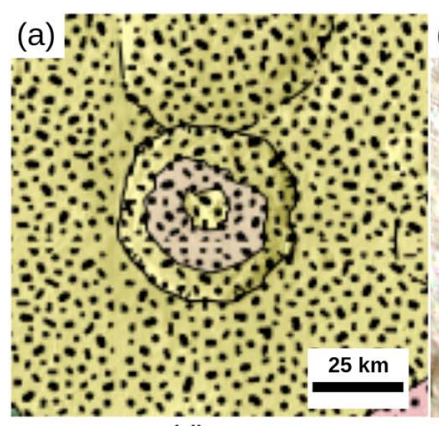

(d)

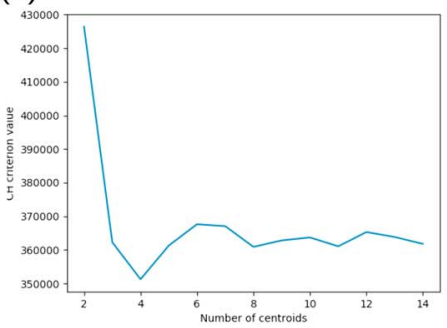

(b)

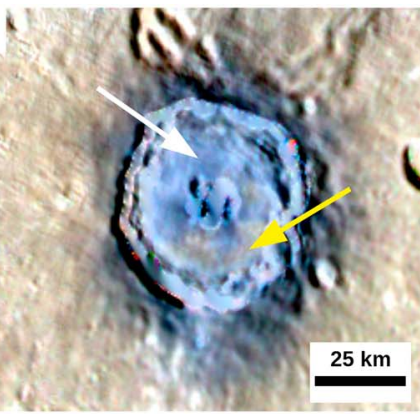

(e)

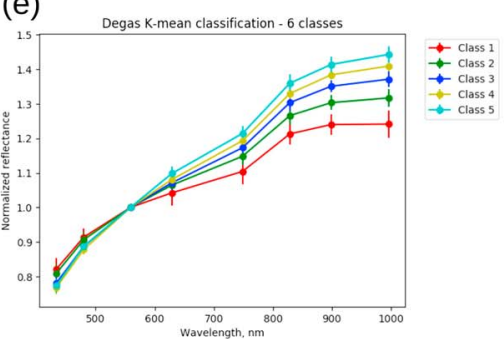

Figure 5. (a) Crop of the geological map around the Degas crater presented in Figure 1 (Guzzetta et al., 2017). The well-preserved crater material is in yellow (c3), the beige corresponds to the smooth crater floor material, and the black dots texture the light colored ejecta. The floor of Degas is mapped by a single geological unit; (b) red-green-blue (996, 749 , and $433 \mathrm{~nm}$ ) of the same region of interest. Red range: $0.06062-0.11039$; green range: $0.0489-0.09464$; blue range: 0.02790-0.05954. The white and yellow arrows point the different terrains identified thanks to the K-mean classification. (c) K-mean classification of our region of interest. (d) Calinski-Harabasz criterion as a function of the number of K-mean clusters. The maximum is reached for six clusters. (e) Average spectra of each K-mean class (except Class 6, which corresponds to the crater shadows).

on the full range of wavelengths covered by MDIS (visible and near infrared). According to equation (1), the bright tones in Figure 4a highlight the terrains with a high global spectral slope (redder). Conversely, the dark tones on Figure 4a are the terrains with a bluer spectral slope. It is not surprising to find once again the pyroclastic deposits among the features with the reddest slopes of Shakespeare, neither to notice that the LRM, the ejecta and the rays constitute the terrains with the shallowest slopes of this quadrangle.

Figure $4 \mathrm{~b}$ aims to identify the areas where the spectral curvature at $600 \mathrm{~nm}$ is the strongest. Our map is similar to the one obtained by Klima et al. (2018), with the highest values of $S C_{600}$ around Degas, Akutagawa, and Sholem Aleichem craters, and in the area located to the south of Sobkou Planitia as well (red circled).

\subsection{Using the Color Combinations}

With the reflectances at 996, 749, and $433 \mathrm{~nm}$ as a color combination (Figure 4c), we enhanced in particular the pyroclastic deposits, bright yellow patches expressing their overall high level of reflectance, yet more pronounced in the longer wavelengths than the average terrains; that is, they are spectrally red; the LRM, local dark blue spots revealing spectral properties in contrast with the pyroclastic deposits, that is, low reflectance and blue spectrum; and the ejecta and rays, white patches and lines, respectively, evidencing their brightness and average spectral slope. IcP are also well visible, like those surrounding Sobkou Planitia. They define a dark gray corona around these plains.

As the first RGB (Figure 4c) map, the RGB map that combines PC2, PC1, and the 433-nm/996-nm ratio (Figure 4d) enhances the pyroclastic deposits (intense yellow); the LRM (intense dark blue); and the bright ejecta and rays of fresh material (white or cyan according how much they are bright compared to how blue is their spectrum) are here even more enhanced (see Figure 4d). The same spectral properties are highlighted in both maps, yet in a clearer way. However, with this color combination, the hollows are also well evidenced in cyan, indicating how bright and blue they are compared to the surrounded areas. Finally, the Caloris smooth plains material is also highlighted with orange and greenish tones, characteristic of the moderate brightness and high spectral slope of this region.

When using $S C_{600}$, the spectral parameter to investigate the presence of the spectral curvature at $600 \mathrm{~nm}$, we obtained the $\mathrm{RGB}\left(\mathrm{R}=S C_{600}, \mathrm{G}=996 / 433 \mathrm{~nm}\right.$, and $\left.\mathrm{B}=1 / S C_{600}\right)$ map shown in Figure 4e. Three kinds of 

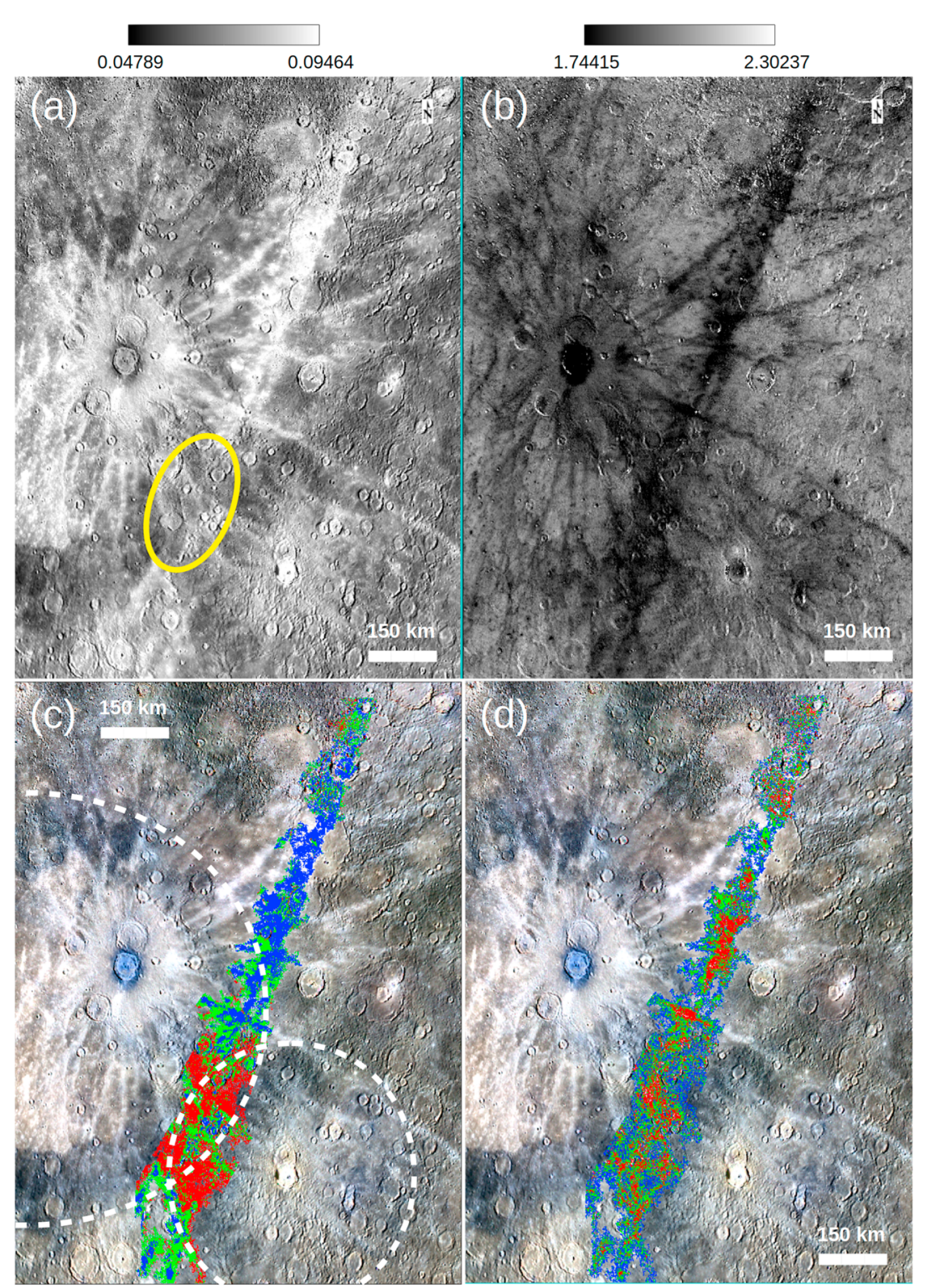

(e)

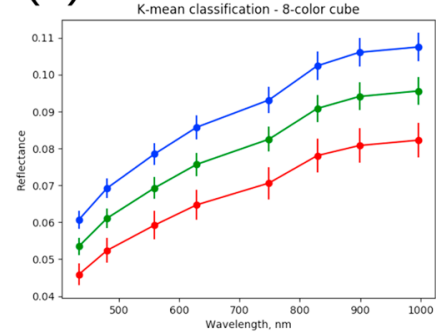

(f)

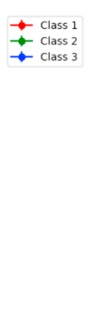

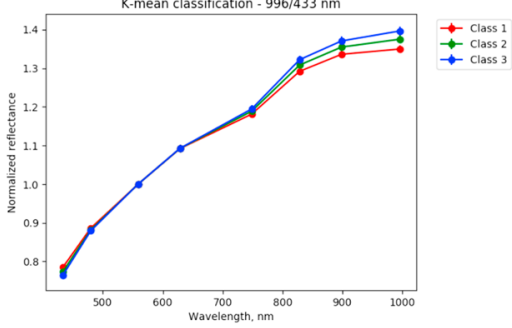

Figure 6. (a) Global view of the OR on the 749-nm (monochrome) map. The yellow oval circles the location where the OR seems to stop. (b) Global view of the OR on the 996/433-nm map. (c) The three classes of the OR overplotted on a RGB (996, 749, and $433 \mathrm{~nm}$ ) map for the K-mean clustering applied on the eight-color cube. Red range: 0.06062-0.11039; green range: 0.0489-0.09464; blue range: 0.02790-0.05954. The dashed lines estimate roughly the extension of IcP surrounding Sobkou bassin and Erté crater. (d) The three classes of the OR overploted on a RGB (996, 749, and $433 \mathrm{~nm}$ ) map for the K-mean clustering applied on the ratio (996/433 nm) mosaic. Red range: 0.06062-0.11039; green range: 0.0489-0.09464; blue range: 0.02790-0.05954. (e) The average spectra of each class identified for the K-mean clustering applied on the eight-color cube. (f) The average spectra of each class identified for the K-mean clustering applied on the ratio $(996 / 433 \mathrm{~nm})$ mosaic. The error bars are within the points. OR $=$ orthogonal ray; RGB = red-green-blue. 
area can be identified: the green areas, which have a global high spectral slope (visible with near-infrared) like pyroclastic deposits and HRP; the blue areas, where the curvature at $600 \mathrm{~nm}$ is low; and the red areas with a likely high curvature at $600 \mathrm{~nm}$. We note that the green areas are scattered over the whole quadrangle, while the most evident blue areas are mainly located to the north of the quadrangle. In this sector of the quadrangle, there are two unnamed Kuiperian craters centered at $62.9^{\circ} \mathrm{N},-101.4^{\circ} \mathrm{W}$ and $64.5^{\circ} \mathrm{N},-104.6^{\circ} \mathrm{W}$ (yellow arrows on Figure 4e), whose ejecta appear particularly blue on this map. The Degas crater, the OR and the terrains containing LRM appear instead as red areas.

After replacing the ratio $433 / 996 \mathrm{~nm}$ in the RGB map of Figure $4 \mathrm{~d}$ by the parameter $S C_{600}$, we obtained a RGB map (Figure 4f) which is very similar. This is not completely surprising because the formula used to calculate $S C_{600}$ (equation 2) is a ratio of reflectances, like for the blue channel of Figure 4d. However, using $S C_{600}$, we have a direct access to the strength of the spectral curvature across the quadrangle: as in Figure 4b, the LRM (blue) seems to be essentially present around Degas, Akutagawa, and Sholem Aleichem craters, and around Sobkou Planitia. However, Figure $4 \mathrm{f}$ is significantly different on the last point: indeed, according to this map, LRM seems to be more or less all around Sobkou Planitia, whereas it was less evident on Figure 4b where LRM appears to be concentrated in the south of Sobkou Planitia. Figure $4 \mathrm{f}$ is thus the best map to identify the LRM: The LRM boundaries are well distinguishable while giving a direct access to the spectral curvature at $600 \mathrm{~nm}$, which is related to the amount of material present.

\subsection{Using the K-Mean Clustering}

We applied a K-mean clustering (see section 3.3 for more details on this method) to two subsets of the eight-color cube of Shakespeare: the Degas crater and the OR. The reader is referred to section 5 for an explanation of these choices.

The analysis of the crater Degas is summarized in Figure 5. We considered an eight-color cube centered on Degas. Six clusters have been identified for this region of interest (see Figure 5d). Class 6, which corresponds to the shadows, is not significant for our analysis. Almost all the floor of Degas is classified as class 1 , whose average spectrum is the shallowest and has the strongest spectral curvature (see Figure $5 \mathrm{e}$ ). Thus, the main material hosted by this crater is most probably the freshest of the studied area. Classes 2 and 3 identify the LRM excaved by the impact.

Regarding the geology, it is also interesting to notice that the smooth crater floor material seems to be isotropically distributed around the central peak (see Figure 5a), while the RGB map shows an anisotropic distribution of this material (see Figure 5b). Our classification (see Figure 5c) confirms this observation and shows that at least two kinds of material characterized by different spectral slopes, hence with probably different mineralogical compositions, cover the floor of Degas. Small hollows have been reported by Blewett et al. (2013) and Thomas et al. (2014) on Degas crater floor but have neither been mapped from a geological point of view (see Guzzetta et al., 2017, and Figure 5a) because they are too small structures, neither in our color maps (e.g., Figure 5b) because of the still too limited spatial resolution. These observations made possible by the K-mean clustering analysis are complementary of the considerations raised with the thresholding analysis, which were done at a quadrangle scale (see section 4.1).

The analysis of the OR is presented in Figures 6 and 7. A (partly supervised, i.e., without using the algorithm of Marzo et al., 2006, because the probed area is too large) K-mean classification applied on this region of interest (ROI hereafter) permits to identify three classes, separated from each other by 0.01 in reflectance (Figures $6 \mathrm{c}$ and 6e). Looking only with the reflectance at $749 \mathrm{~nm}$ (Figure 6a), the OR seems to stop before the location circled on the figure with the yellow oval, showing a global reflectance gradient from south to north. However, if we consider the ratio 996/433-nm map (Figure 6b), it is obvious that the OR runs continuously from north to south of the view, showing no peculiar trend for the spectral slope (Figures $6 \mathrm{~d}$ and $6 \mathrm{f}$ ). Yet, if we look closer to Figure $6 \mathrm{c}$, it seems that the OR becomes brighter at its very end (south). Moreover, the two locations identified as Class 1 are likely due to Sobkou basin and/or Erté crater underlying IcP, since these terrains perfectly overlap the red areas (see dashed lines on Figure 6c).

Two different stretchings of the same RGB (996-749-433 nm) have been applied. On the top right of Figure 7; the stretching highlights in particular the Hokusai ray, even the darkest part, which appears in dark blue. In addition, five small ROIs have been selected at different locations alongside, but outside the OR: three are on the SP and two on the IcP. The average spectra of these ROIs (bottom of Figure 7) clearly indicate that the SP underlying the OR are 0.01 higher in reflectance than the IcP underlying the OR. 

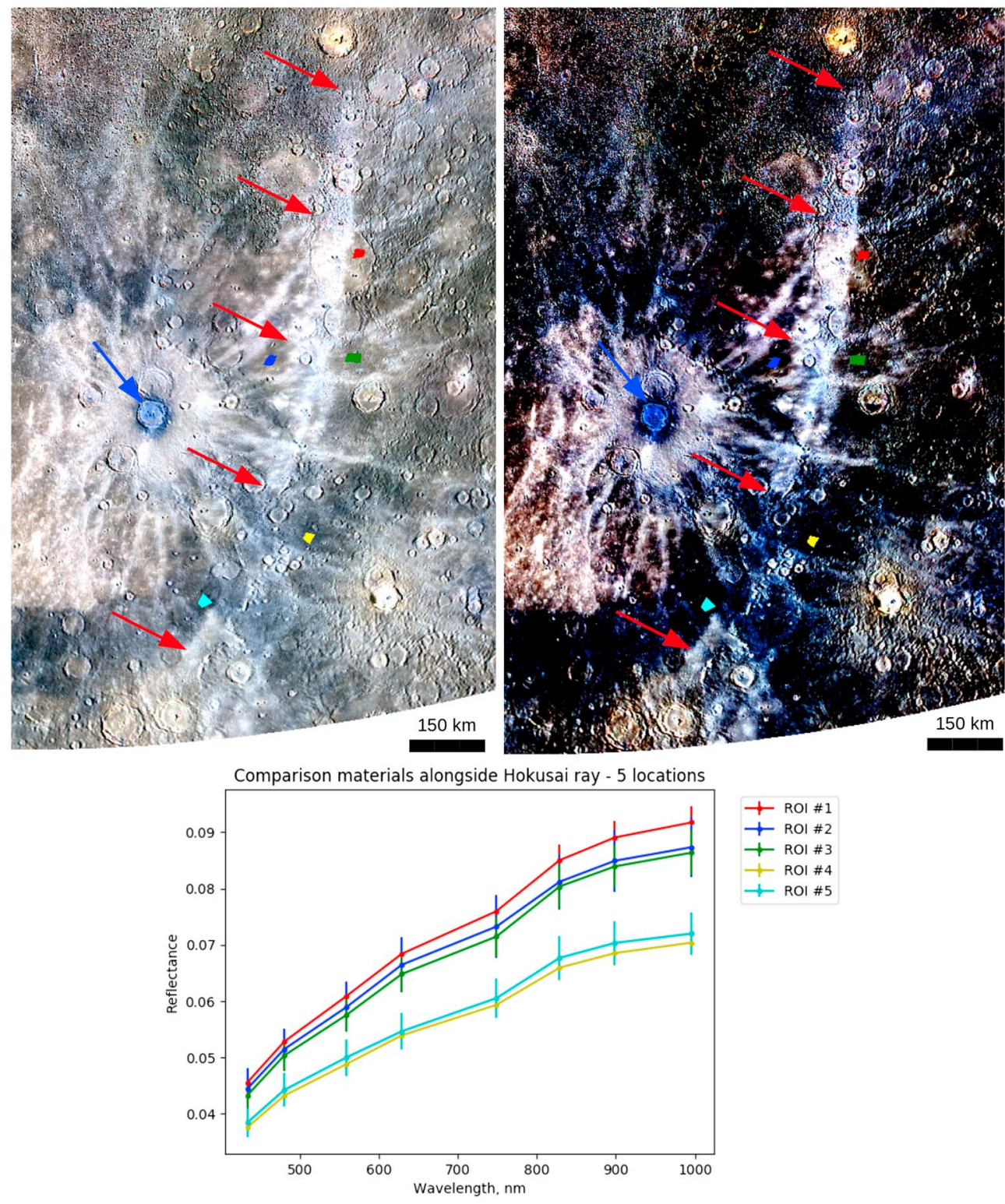

Figure 7. Top: Two global views in Lambert conformal conic projection of the orthogonal ray (red arrows) on the same red-green-blue (996-749-433 nm) map with different stretchings, together with five selected regions of interest (red, blue, green, yellow, and cyan polygons). The blue arrow denotes the Degas crater. Top left: Red range: 0.06254-0.11021; green range: $0.04946-0.09450$; blue range: $0.02909-0.05932$. Top right: Red range: $0.08820-0.11021$; green range: 0.07464-0.09450; blue range: 0.04480-0.05932. Bottom: The average spectra of the regions of interest (ROI).

\section{Discussion}

The results obtained from the different approaches and described in section 4 permit to highlight spectral variations that can be related to compositional variations at quadrangle scale, with two examples of ROIs. In the following section, we summarize which units can be clearly separated. If not precise, the images presented are subsets of maps in equirectangular projection.

\subsection{LRM Alteration}

Numerous laboratory experiments have been conducted to investigate the effects of space weathering on the spectral properties of airless bodies of our solar system like asteroids. Most commonly, space weathering produces a reddening and a darkening of the surface (Brunetto et al., 2015). However, it has been shown that these effects are not systematic for every asteroid class but depends also on the initial albedo (Lantz et al., 2015). Thus, the darkest C-type asteroids (the richest in carbon) can have the opposite spectral behavior 
with respect to space weathering, that is, bluing and brightening. Yet, these asteroids and their associated meteorites have an albedo (e.g., $\simeq 5 \%$ at $550 \mathrm{~nm}$ for the Murchison meteorite; Lantz et al., 2015) lower than the albedo of LRM on Mercury ( $\simeq 13 \%$ at $550 \mathrm{~nm}$ for the Degas crater, $\simeq 8 \%$ at $550 \mathrm{~nm}$ for the Basho crater, which is surrounded by LRM darker than around Degas, like are Akutagawa and Sholem Aleichem craters; Denevi \& Robinson, 2008). The albedo of LRM is closer to the one of the Allende meteorite ( $~ 11 \%$ at $550 \mathrm{~nm}$; Brunetto et al., 2014) for which the space weathering weakly reddens and darkens the surface. Nevertheless, LRM on Mercury probably contains carbon in higher quantity than in the carboneous meteorites (up to $\simeq 4 \%$ wt. for LRM; Klima et al., 2018, $\simeq 2 \%$ wt. for Murchison and $\simeq 0.3 \%$ wt. for Allende; Lantz et al., 2015). Besides, the Tagish Lake meteorite, which is darker (albedo between $\simeq 2.0$ and $3.5 \%$ at $550 \mathrm{~nm}$ ); (Izawa et al., 2015) and contains even more carbon than LRM (5.5\% wt. (Lantz et al., 2015)), becomes brighter and bluer after irradiation. Therefore, the LRM of Mercury is an intermediate case between the cases of Murchison meteorite and Tagish Lake meteorite and thus can darken and redden when exposed to the space weathering.

The physical mechanisms that occur when LRM is exposed to space weathering could vary, depending on the albedo of the LRM. According to Brunetto et al. (2015), for bright material like Allende meteorite (or brighter LRM in our case), the mechanisms can be ordinary chondrites like, that is, the formation of nanosized particules of iron; for darker material such as Tagish Lake (or darker LRM in our case), the mechanisms may be terrestrial bitumens-like, that is, the formation of $\mathrm{C}-\mathrm{H}$ bonds and the increasing of aromaticity. Nevertheless, we can not exclude that the differences between Mercury and carboneous chondrites (FeO content, mineralogical differences such as the presence of hydrated silicates and oxidized material, e.g., magnetite) could also cause carboneous chondrites response to space weathering to differ from that of Mercury.

\subsection{Global Lithology of Shakespeare}

Looking at PC2 (Figure 2) and its Class 1 average spectrum, we can claim that Degas has the shallowest spectrum of the Shakespeare quadrangle. Even if we can not exclude that the rocks composition plays a significant role in this spectral property, it means above all that it is most probably the youngest feature of the area. This would explain why its rays system is so well preserved. Moreover, on the same map, the three main spots containing LRM fall in the same class (Class 2). However, Figure 3 allows us to see that the LRM around Degas is brighter compared to the LRM associated with the Akutagawa and Sholeim Aleichem craters. The explanation of this difference could be related to the age of the material involved. Indeed, to explain the brightness variability of the hollows in the Dominici crater, Vilas et al. (2016) proposed that these hollows could have different ages or could be at different evolution states, for example, could have undergone space weathering processes for a different period of time.

Following the considerations addressed in section 5.1, LRM could darken while aging. This confirm that the LRM around the Degas crater is the youngest of the three LRM spots. (Domingue et al., 2014) raised the possibility that (at least) the implantation of hydrogen ions in Mercury's soil can be at saturation level. Assuming this hypothesis for the majority of the Mercurian surface, we can raise a constraint on the time scale of this process on Mercury. Guzzetta et al. (2017) mapped the ejecta of the Akutagawa crater in the same unit than the ejecta of the Degas crater (c3, well-preserved crater material; see Figure 1), while the ejecta of the Sholem Aleichem crater were mapped in another unit (c2, degraded crater). According to the geological time scales of Mercury (e.g., see the lower right corner of Figure 1), the Akutagawa crater could have formed during the Mansurian or at the beginning of the Calorian, whereas the Sholem Aleichem crater probably formed at the end of the Calorian or at the beginning of the Tolstojan. Yet, the Akutagawa and Sholem Aleichem craters are in the same class of reflectance (see Figure 3). This means that the saturation in terms of space weathering of the surface of Mercury should be reached in a relatively short geological time, that is, during the Mansurian age (between -1 and $-3.6 \mathrm{Gyr}$ ). This is in adequation with the definition of the beginning of the Mansurian, from which craters have no visible rays system. Besides, it is interesting to notice that the rate of optical maturation on Mercury has been estimated to be about four times faster than on the Moon (Braden \& Robinson, 2013), where large rayed craters reach optical maturity after 800-1000 Myr (Hawke et al., 2004). Mercury rays should thus reach maturity after only 200-250 Myr, so much before the beginning of the Mansurian. This difference between beginning of the Mansurian and estimated optical maturity can be explained by the difference in mineralogy between Mercury and the Moon. Indeed, the Mercurian crust is dominated by mafic minerals with low abundance in iron, whereas the lunar crust contains mainly Fe-rich minerals. These minerals are structurally different, therefore they can have a different behavior (e.g., lower efficiency, so acting at longer timescales) when exposed to space weathering. Notwithstanding the above considerations, the hypothesis that LRM darkens when aging is not always consistent with 
observations on a global scale of the planet, as previously suggested by Murchie et al. (2015): The LRM is darker in the younger craters, suggesting that it is gardened with age into the regolith and becomes less concentrated. In addition, Moroz et al. (2004) showed the irradiation of hydrocarbon materials tends to reduce their high spectral slope and to slightly increase their reflectance in the visible. A difference in the content of darkening agent seems thus the most plausible. This would indicate the presence underneath the surface of a nonhomogeneous graphite crust (Peplowski et al., 2016; Vander Kaaden \& McCubbin, 2015) at each point.

Since Hokusai is a Kuiperian crater (Banks et al., 2017), its rays have not been weathered a lot and are thus still fresh. As the common effects of the space weathering are the darkening and the reddening of a surface (Domingue et al., 2014; Brunetto et al., 2015; Pieters \& Noble, 2016), it is therefore reasonable to expect that the OR in Shakespeare is characterized by a spectral slope bluer than the average terrains of Mercury. This assumption is confirmed by the ratio 996-nm/433-nm map (Figure 4a), where the dark features, for example the OR, have a shallower slope, so are expected to be fresher than the surrounding terrains. However, the thresholding of the reflectance (Figure 3) permitted to identify a decrease in reflectance at the end of the OR. This part of the quadrangle corresponds to the darkest part of the OR in our quadrangle (see section 5.3.2 and in particular Figure 6). This could suggest that the material excaved from the depth of Degas contained LRM and has mixed with the fresh material of the OR, thus making it darker in this area.

Based on global studies of pyroclastic deposits, it has been hypothesized that their spectral characteristics (high reflectance and high spectral slope) are due to compositional or physical properties, rather than age. According to Kerber et al. (2011) who relied on the works of Lucey et al. (1995) for the Moon, the brightness and redness of the Mercurian pyroclastic deposits can be explained by their low-iron content compared to the surrounding terrains, which are already poor in iron compared to the Moon surface for example. Moreover, the same authors put forward that the material which composes the pyroclastic deposits did not crystallize a large amount of opaque minerals, e.g. sulfides. This is in agreement with Nittler et al. (2014) who showed that the largest pyroclastic deposit of Mercury (Rachmaninoff) is sulfur depleted. Finally, Besse et al. (2015) demonstrated that the grain size variability of the pyroclastic deposits material can impact their spectral properties: the further from the pyroclastic vent, the smaller the grain size is, and the less red the pyroclastic deposit (such considerations can be extended to craters ejecta like in D'Incecco et al., 2016). Our results statements are consistent with these statements. Indeed, on the same map discussed above (Figure 4a), pyroclastic deposits have high values of ratio $996 / 433 \mathrm{~nm}$, so have the highest spectral slope of the quadrangle. The yellow color of the pyroclastic deposits in Figure $4 \mathrm{~d}$ is also indicative of a bright material with a redder spectral slope, since with high values for the green and red channels, respectively, get yellow pixels. Besides, the cyan color of the OR is consistent with of a bright bluer material leading to high values to the green and blue channels, respectively.

On the first RGB using $S C_{600}$ (Figure 4e), we noticed that there are spectrally reddish (with high spectral slope, i.e., with green tones on the map) terrains both on the eastern and the western parts of Shakespeare. This spectral property can be due either to the terrains maturity or to a difference in composition. Looking at the cratering rate of both parts (see Figure 1), the eastern part appears more craterized than the western part. With the relation to the time of surface exposure to space weathering processes, we can thus consider it composed of more mature terrains. However, more recent terrains outcrop in the western part. These terrains are ejecta of the Caloris basin and smooth plains, which could be naturally redder than the average of Mercury. Moreover, on the same map, we can see that the OR appears in red, so has a high spectral curvature. Nevertheless, since this feature has a very high reflectance (see Figure 3), we cannot interpret the high curvature to the presence of LRM. Looking at the formula of $S C_{600}$ (equation (2)), it could be rather a bias effect due to the global high level of reflectance of the OR. We can notice the same interpretation is valid for the Degas rays. Finally, we pointed out that the ejecta of the two Kuiperian craters in the north of the quadrangle (yellow arrows on Figure 4a) are blue in contrast to the Degas crater. This shows that these ejecta have a very low spectral curvature, thus do not contain LRM. Therefore, the lithology of these two unnamed craters is different from the lithology of Degas.

We saw in Figure $4 \mathrm{~b}$ that the highest values of $S C_{600}$ are concentrated around the Degas, Akutagawa, and Sholem Aleichem craters, and in the south of Sobkou Planitia. However, we can not conclude that all the terrains highlighted on this map contain LRM. Indeed, according to the geological map of Guzzetta et al. (2017), the terrains surrounding Sobkou Planitia are IcP and there is no reason for the presence of LRM only (or more) concentrated in the south of Sobkou Planitia. This might be a hint of lithology heterogeneities for 
the IcP unit: the spectral properties of the IcP in the south of Sobkou Planitia seem different from the other IcP in the quadrangle. The IcPs rich in LRM surrounding Sobkou Planitia could therefore represent a subunit of the more widespread, strictly speaking IcP or their specific facies. Using $S C_{600}$ as the spectral parameter for the blue channel instead of the ratio 433/996 nm in Figure 4d, we have to qualify this statement, as it appears that the LRM is mainly localized in the south of Sobkou Planitia, even if we cannot exclude the presence of LRM in smaller quantity all around this basin. Hence, the color mapping can help solve such kind of inconsistencies, and thus the precise lithological nature of rocks, which look similar in the first instance.

From what precedes, we can identify the following spectral units, characterized by specific spectral properties:

- The LRM spots, with a low reflectance (Figure 3), a bluer spectral slope than the average, that is, smaller values of PC2 (Figure 2) but higher values of 433/996 nm (Figure 4d), and a high spectral curvature (Figures 4b, 4e, and 4f).

- The OR, presenting a high reflectance (Figure 3) and a bluer spectral slope than the average (Figures 2 and 4a).

- The Degas crater, whose floor has a quite high reflectance (as high as the SPs of Sobkou Planitia; see Figure 3) and the shallowest spectral slope of the quadrangle (Figures 2 and 4a).

- The IcP, mainly pointed out thanks to their quite low spectral slope (Figure 2), and to their high spectral curvature for the ones which are rich in LRM (Figure 4b). They also have a low reflectance (Figure 3), but higher than the LRM.

- The Caloris material on the western of the Shakespeare quadrangle, with a high (Odin formation) or very high (Caloris plains) reflectance (Figure 3) and a spectral slope similar to the average terrains.

- Lastly, the terrains covered by the pyroclastic deposits, showing a very high reflectance (Figure 3) and a very high spectral slope (Figure 2).

Furthermore, the central region of Shakespeare, encompassing Sobkou Planitia, the Degas crater and the OR are probably relevant choices to probe the diversity of the global lithology of Mercury.

\subsection{Local Lithology of Shakespeare: The Examples of Two Regions of Interest} 5.3.1. The Degas Crater

The Degas crater $(\mathrm{D} \approx 53.5 \mathrm{~km})$ located at $37.1^{\circ} \mathrm{N},-137.2^{\circ} \mathrm{W}$ within Sobkou Planitia is also a very interesting target for BepiColombo. It has been referred to the Kuiperian period (started nearly $280 \pm 60$ Myr ago; see Banks et al., 2017; Xiao et al., 2014), because this crater shows a well-preserved important system of bright rays, well developed in length, and even clearly visible on monochomatic base maps. Further, whereas this crater presents no specificity on the geological map of Guzzetta et al. (2017), it is very interesting from a spectral point of view for two reasons: (1) Compared to the other craters in the quadrangle, its floor appears in distinctive blue tones in the color-composite maps of Figures 4c, 4d, and 4f; (2) there is LRM visible on the proximal ejecta surrounding the crater rim, thus giving a hint of the lithology of this area.

There can be two interpretations of the classification obtained (see section 4.3): either the material outside the crater has not the same composition than the material which is on the floor or it is the same material which underwent different processes, making it evolving differently (or both).

Moreover, two spectrally different terrains have been evidenced in the floor of Degas. The one which is very blue on the RGB map (see white arrow in Figure 5b) could corresponds to a material with a high content in fresh darkening agent excaved from depth during the impact. The second could be the same material but mixed with an older material like the Sobkou Planitia smooth plains material, which would explain the different spectral slope of the different terrains. Here, the K-mean clustering helped us to highlight the lithological heterogeneities (two classes associated to two terrains) of Degas floor. This was not possible with the thresholding analysis (only one class).

The blue color of the terrains classified as Class 1 in Figure 5c can be a reminder of some craters in the Caloris basin, which also have a blue floor like Degas (Ernst et al., 2015; Potter \& Head, 2017). However, the floor of these craters are blue only with the color combination using PC2, PC1, and the ratio $433 / 996 \mathrm{~nm}$. In addition, with this color combination, the blue is sightly different for Degas floor on the one hand, and for the LRM surrounding Degas and the floor of craters in Caloris on the other hand. Thus, there are three specific cases: (1) it is the same material but in different quantities; (2) it is the same material which evolved in different ways; (3) or they correspond to different materials. The most probable is the first case, as this 
would explain that Degas floor is brighter than the surroundings of the crater. Indeed, the thresholding of the reflectance at $749 \mathrm{~nm}$ (Figure 3) separates the floor of the crater (class 2) and its surroundings (Class 3). Moreover, this would be consistent with the thresholding of PC2 (Figure 2) for which the floor and the surroundings of Degas are in a unique class (Class 1). This way, the darker surroundings of Degas would contain more LRM than the floor. The last case is also quite possible, since both areas could contain LRM but more or less mixed with other materials which have different spectral properties (e.g., SP).

\subsubsection{The Hokusai Ray}

Another ROI can be the OR (see Figure 7, red arrows). This feature extends nearly from $33.3^{\circ} \mathrm{N},-121.1^{\circ} \mathrm{W}$ to $49.3^{\circ} \mathrm{N},-112.2^{\circ} \mathrm{W}$ and is estimated to be $\approx 750 \mathrm{~km}$ long. According to Xiao et al. (2016) and Ernst et al. (2018), the OR comes from the Hokusai crater $\left(57.8^{\circ} \mathrm{N}, 16.6^{\circ} \mathrm{W}\right)$. This big (diameter: $\left.114 \mathrm{~km}\right)$ Kuiperian crater has the most widespread system of rays on Mercury and is $\sim 3,516 \mathrm{~km}$ far from the Degas crater. The ray we are interested in is long (several thousand of kilometers) and particularly large (about 50-km width) compared to the other rays of Hokusai (about 20-km width).

The K-mean classification of the OR has evidenced three well separated classes of reflectance, with the darkest part in the southern part of the ray but not at its very end (see section 4.3 and Figure 6). The 996/433-nm ratio map shows that this darkest part can not correspond to a discontinuity of the ray. This statement is confirmed if one stretches the RGB map (see top left of Figure 7): The darkest part of the OR appears in dark blue. This can be interpreted as a difference in composition, probably resulting in the competition of two effects:

1. A decrease of the amount of fresh material from north to south. This is consistent with the location from where the ray originates, that is, the Hokusai crater, and passing close to the north pole. Thus, the more southward we are, the less material there should be, and so the darker the ray appear. This would also explain the global gradient in reflectance.

2. An influence of the reflectance of the underlying terrains. The IcP surrounding the Sobkou bassin and/or the Erté crater centered on $27.4^{\circ} \mathrm{N},-117.3^{\circ} \mathrm{W}$, in the southeast of the ray (see dashed circles in Figure 6) are much darker than the SP in the northern part of the OR. Thus, the much brighter material ejected from the Hokusai crater may have mixed with the darker material around Sobkou and/or Erté, consistently decreasing the reflectance of this part of the ray.

\section{Conclusions}

The use of high-resolution color maps spatially correlated with each other is essential to build a geological map taking into account both the morphology and the composition of the Mercurian surface. In this paper, we presented a high-resolution eight-color mosaic of the Shakespeare quadrangle. This product constitutes a good starting point to study the general spectral and lithological properties of this region of the north hemisphere of Mercury. Several examples have been given, among which the presence of high quantities of LRM mainly around Degas, Akutagawa, and Sholem Aleichem, and in the south of Sobkou Planitia and the highlighting of intrinsically redder material (HRP) in the western part of Shakespeare. Then, we focused on some areas which could present a high interest for the future BepiColombo mission: the Degas crater and the main Hokusai ray. What emerges from this analysis is that Mercury had a past-and may have an ongoing-activity that brings to light the complexity of its crust, and more generally of its interior.

The main spectral units identified are the following, with their values for the spectral parameters (which will be useful for future works like the integration of the spectral units to the geological map of the Shakespeare quadrangle) summarized in Table 5:

- The LRM spots, with a low reflectance and a bluer spectral slope than the average (smaller values of PC2), and for which we evidenced a higher concentration around Degas, since LRM (Murchie et al., 2015) and more globally hydrocarbon materials (Moroz et al., 2004) slightly brighten when aging.

- The OR, presenting a high reflectance and a bluer spectral slope than the average. We showed this ray is not homogeneous, at least in terms of reflectance, and that it may be a hint of the underlying lithology of the surface, since the ray-forming material ejected from Hokusai has probably reworked the local material and may also be affected by underlying IcP around Sobkou and/or Erté.

- The Degas crater itself, whose floor has a quite high reflectance (as high as the SP of Sobkou Planitia) and the shallowest spectral slope of the quadrangle. Its ejecta contain LRM, which is bluer than the average 
Table 5

Values of the Spectral Parameters for Each Spectral Unit

\begin{tabular}{|c|c|c|c|c|c|c|c|c|}
\hline \multirow[b]{2}{*}{ Spectral unit } & \multicolumn{2}{|c|}{ R749 } & \multicolumn{2}{|c|}{$\mathrm{PC} 2$} & \multicolumn{2}{|c|}{$996 / 433$} & \multicolumn{2}{|c|}{$S C_{600}$} \\
\hline & Min & Max & Min & Max & Min & Max & Min & Max \\
\hline LRM & 0.035 & 0.058 & -0.01 & -0.005 & 1.64 & 1.86 & 0.015 & 0.05 \\
\hline OR & 0.085 & 0.102 & -0.01 & -0.005 & 1.64 & 1.86 & \multicolumn{2}{|c|}{$<0.015$} \\
\hline Degas & \multicolumn{2}{|c|}{$\mathrm{X}$} & -0.022 & -0.01 & 1.35 & 1.64 & & \\
\hline $\mathrm{IcP}$ & \multicolumn{2}{|c|}{$\mathrm{X}$} & -0.005 & -0.001 & \multicolumn{2}{|c|}{$\mathrm{X}$} & \multicolumn{2}{|c|}{$<0.015$} \\
\hline Caloris & 0.069 & 0.085 & 0.0035 & 0.007 & \multicolumn{2}{|c|}{$\mathrm{X}$} & \multicolumn{2}{|c|}{$<0.015$} \\
\hline $\mathrm{PD}$ & 0.085 & 0.102 & 0.007 & 0.018 & 2.20 & 2.40 & \multicolumn{2}{|c|}{$<0.015$} \\
\hline
\end{tabular}

Note. $\mathrm{R} 749=$ reflectance at $749 \mathrm{~nm} ; \mathrm{PC} 2=$ principal component $2 ; S C_{600}=$ spectral curvature around $600 \mathrm{~nm} ; \mathrm{LRM}=$ low reflectance material; OR = Hokusai orthogonal ray; IcP = intercrater plains; Caloris $=$ Caloris material $\mathrm{PD}=$ pyroclastic deposits. $\mathrm{X}=$ no value (not a separated unit).

terrains. This confirms that its own bluer color is not only due to its youth, but also to its composition. The smooth plains floor also seems to be partly covered by LRM, but not completely.

- The IcP, pointed out thanks to their quite low spectral slope. They also have a low reflectance, but higher than the LRM. However, we put forward that the spectral properties of the IcP in the south of Sobkou Planitia are different from the other IcP in the quadrangle. These IcP are rich in LRM and could therefore represent a subunit of the more widespread IcP in the strict sense of the term, or their specific facies. This is a good indication in favor of a heterogeneous primary crust for Mercury, at least in this location, with a bigger concentration of graphite in the south of Sobkou Planitia than in the north.

- The Caloris material on the western of Shakespeare, with a high (Odin formation) or very high (Caloris plains) reflectance and a spectral slope similar to the average terrains.

- Lastly, the terrains covered by the pyroclastic deposits, showing a very high reflectance and a very high spectral slope, consistent with their volcanic nature.

The analysis of our identified ROIs was based on an algorithm that computes the natural number of clusters to classify them. We analyzed the spectral properties of the OR and the Degas crater. In particular, we saw that the floor of Degas is covered by a bluish material, which is observed nowhere else, at least in the Shakespeare quadrangle. However, (Guzzetta et al., 2017) mapped Degas with the same geological unit than the surrounding craters (e.g., Erté) which do not present such kind of deposits on their floors.
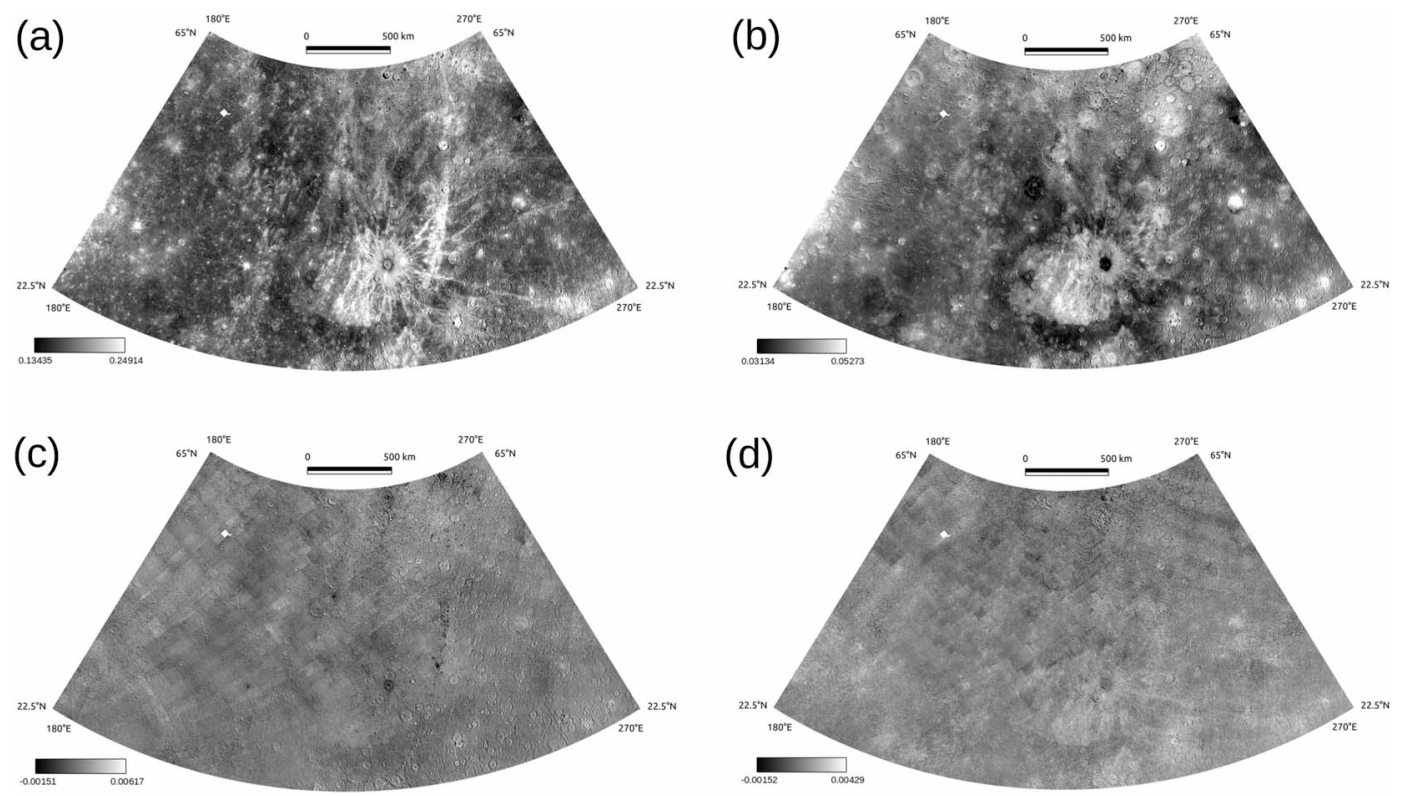

Figure A1. Panel of maps showing the four first principal components of the Shakespeare quadrangle. (a) PC1; (b) PC2; (c) PC3; (d) PC4. 
This discrepancy between the geological map of Guzzetta et al. (2017) and our color map can justify to define a spectral (or lithological) subunit, to combine both morphological and compositional information given by the two maps. In the same perspective, a closer study of the region in the south of Sokbou, which seems to contain more LRM than in the north of this basin, can be highly interesting. This would require to understand how Sobkou has been formed, so draw its chronology.

The final goal of the geological mapping campaign is to obtain a global series of 1:3M-scale geological maps of Mercury in support to the ESA/JAXA BepiColombo mission (launched on 19 October 2018), in order to set up the context for mission operations and help to redefine mission goals. Notably, these cartographic products will be useful for SIMBIO-SYS, one of the 11 instruments onboard Mercury Planetary Orbiter (under the supervision of ESA), which will take high-resolution images of the surface of the planet.

\section{Appendix A: The Four First Principal Components of the Shakespeare Quadrangle}

In Figure A1 are shown the mosaics of the four first PCs of the Shakespeare quadrangle. As a reminder, PC1 gives information on the brightness variations, while PC2 shows spectral variations mainly related to the spectral slope of the continuum. PC3 and PC4 have no real physical meaning.

\section{Acknowledgments}

This work was partly supported by the Centre National d,Études Spatiales, the Agenzia Spaziale Italiana, and the SIMBIO-SYS project (ASI-INAF 2017-47-H.0). C. Carli and F. Zambon were funded by the European Union's Horizon 2020, research grant agreement 776276-PLANMAP. D. Perna received funding from the European Union's Horizon 2020 research and innovation programm under the Marie Sklodowska-Curie grant agreement 664931. The authors want to thank G. Marzo and T. Roush for having kindly provided the tools for the K-mean analysis. Lastly, the authors thank two anonymous reviewers for their careful and constructive reviews. The PDS images used in this work are available on https://pds-imaging.jpl.nasa.gov/ Atlas/ and were processed thanks to ISIS, a software developed and provided by USGS.

\section{References}

Anderson, J. A., Sides, S. C., Soltesz, D. L., Sucharski, T. L., \& Becker, K. J. (2004). Modernization of the Integrated Software for Imagers and Spectrometers, Lunar and Planetary Science XXXV, abstract \#2039.

Banks, M. E., Xiao, Z., Braden, S. E., Barlow, N. G., Chapman, C. R., Fassett, C. I., \& Marchi, S. S. (2017). Revised constraints on absolute age limits for Mercury's Kuiperian and Mansurian stratigraphic systems. Journal of Geophysical Research: Planets, 122, 1010-1020. https:// doi.org/10.1002/2016JE005254

Besse, S., Doressoundiram, A., \& Benkhoff, J. (2015). Spectroscopic properties of explosive volcanism within the Caloris basin with MESSENGER observations. Journal of Geophysical Research: Planets, 120, 2102-2117. https://doi.org/10.1002/2015JE004819

Blewett, D. T., Chabot, N. L., Denevi, B. W., Ernst, C. M., Head, J. W., Izenberg, N. R., et al. (2011). Hollows on Mercury: MESSENGER Evidence for Geologically Recent Volatile-Related Activity. Science, 333, 1856-1859.

Blewett, D. T., Robinson, M. S., Denevi, B. W., Gillis-Davis, J. J., Head, J. W., Solomon, S. C., et al. (2009). Multispectral images of Mercury from the first MESSENGER flyby: Analysis of global and regional color trends. Earth and Planetary Science Letters, 285(3-4), 272-282. https://doi.org/10.1016/j.epsl.2009.02.021

Blewett, D. T., Stadermann, A. C., Susorney, H. C., Ernst, C. M., Xiao, Z., Chabot, N. L., et al. (2016). Analysis of MESSENGER high-resolution images of Mercury's hollows and implications for hollow formation. Journal of Geophysical Research: Planets, 121, 1798-1813. https://doi.org/10.1002/2016je005070

Blewett, D. T., Vaughan, W. M., Xiao, Z., Chabot, N. L., Denevi, B. W., Ernst, C. M., et al. (2013). Mercury's hollows: Constraints on formation and composition from analysis of geological setting and spectral reflectance. Journal of Geophysical Research: Planets, 118, $1013-1032$. https://doi.org/10.1029/2012JE004174

Braden, S. E., \& Robinson, M. S. (2013). Relative rates of optical maturation of regolith on Mercury and the Moon. Journal of Geophysical Research: Planets, 118, 1903-1914. https://doi.org/10.1002/jgre.20143

Brunetto, R., Lantz, C., Ledu, D., Baklouti, D., Barucci, M. A., Beck, P., et al. (2014). Ion irradiation of Allende meteorite probed by visible, IR, and Raman spectroscopies. Icarus, 237, 278-292.

Brunetto, R., Loeffler, M. J., Nesvorny, D., Sasaki, S., \& Strazzulla, G. (2015). Asteroid Surface Alteration by Space Weathering Processes. In P. Michel, F. E. DeMeo, W. F. Bottke (Eds.), Asteroids IV (pp. 597-616). Tucson: University of Arizona. https://doi.org/10.2458/azu_ uapress_9780816532131-ch031

Calinski, T., \& Harabasz, J. (1974). A dendrite method for cluster analysis. Communications in Statistics - Theory and Methods, 3(1), 1-27. https://doi.org/10.1080/03610927408827101

D'Incecco, P., Helbert, J., D'Amore, M., Ferrari, S., Head, J. W., Maturilli, A., \& Hiesinger, H. (2016). A geologically supervised spectral analysis of 121 globally distributed impact craters as a tool for identifying vertical and horizontal heterogeneities in the composition of the shallow crust of Mercury. Planetary and Space Science, 132, 32-56. https://doi.org/10.1016/j.pss.2016.08.004

Davies, M. E., Dornik, S. E., Gault, D. E., \& Strom, R. G. (1978). Atlas of Mercury (https://history.nasa.gov/sp-423/sp423.htm).

Denevi, B. W., Chabot, N. L., Murchie, S. L., Becker, K. J., Blewett, D. T., Domingue, D. L., et al. (2018). Calibration, projection, and final image products of MESSENGER's Mercury Dual Imaging System. Space Science Reviews, 214(1), 52. https://doi.org/10.1007/ s11214-017-0440-y

Denevi, B. W., \& Robinson, M. S. (2008). Mercury's albedo from Mariner 10: Implications for the presence of ferrous iron. Icarus, 197, 239-246.

Denevi, B. W., Robinson, M. S., Solomon, S. C., Murchie, S. L., David, T., Domingue, D. L., et al. (2009). The evolution of Mercury's crust: A global perspective from MESSENGER. Science, 324(5927), 613-618.

Domingue, D. L., Chapman, C. R., Killen, R. M., Zurbuchen, T. H., Gilbert, J. A., Sarantos, M., et al. (2014). Mercury's weather-beaten surface: Understanding Mercury in the context of lunar and asteroidal space weathering studies. Space Science Reviews, 181, $121-214$.

Domingue, D. L., Denevi, B. W., Murchie, S. L., \& Hash, C. D. (2016). Application of multiple photometric models to disk-resolved measurements of Mercury's surface: Insights into Mercury's regolith characteristics. Icarus, 268, 172-203. https://doi.org/10.1016/j.icarus. 2015.11.040

Domingue, D. L., Murchie, S. L., Chabot, N. L., Denevi, B. W., \& Vilas, F. (2011). Mercury's spectrophotometric properties: Update from the Mercury Dual Imaging System observations during the third MESSENGER flyby. Planetary and Space Science, 59, $1853-1872$. 
Domingue, D. L., Murchie, S. L., Denevi, B. W., Ernst, C. M., \& Chabot, N. L. (2015). Mercury's global color mosaic: An update from MESSENGER's orbital observations. Icarus, 257, 477-488. https://doi.org/10.1016/j.icarus.2014.11.027

Ernst, C. M., Chabot, N. L., \& Barnouin, O. S. (2018). Examining the potential contribution of the Hokusai impact to water ice on Mercury. Journal of Geophysical Research: Planets, 123, 2628-2646. https://doi.org/10.1029/2018je005552

Ernst, C. M., Denevi, B. W., Barnouin, O. S., Klimczak, C., Chabot, N. L., Head, J. W., et al. (2015). Stratigraphy of the Caloris basin, Mercury: Implications for volcanic history and basin impact melt. Icarus, 250, 413-429.

Gaddis, L., Anderson, J., Becker, K., Becker, T., Cook, D., Edwards, K., et al. (1997). An overview of the Integrated Software for Imaging Spectrometers (ISIS), Lunar and Planetary Science XXVIII, abstract \#1226.

Goudge, T. A., Head, J. W., Kerber, L., Blewett, D. T., Denevi, B. W., Domingue, D. L., et al. (2014). Global inventory and characterization of pyroclastic deposits on Mercury: New insights into pyroclastic activity from MESSENGER orbital data. Journal of Geophysical Research: Planets, 119, 635-658. https://doi.org/10.1002/2013JE004480

Guzzetta, L., Galluzzi, V., Ferranti, L., \& Palumbo, P. (2017). Geology of the Shakespeare quadrangle (H03), Mercury. Journal of Maps, 13(2), 227-238. https://doi.org/10.1080/17445647.2017.1290556

Hapke, B. (1981). Bidirectional reflectance spectroscopy: 1. Theory. Journal of Geophysical Research, 86(4), 3039-3054. https://doi.org/10. 1029/JB086iB04p03039

Hapke, B., Denevi, B., Sato, H., Braden, S., \& Robinson, M. (2012). The wavelength dependence of the lunar phase curve as seen by the Lunar Reconnaissance Orbiter wide-angle camera. Journal of Geophysical Research, 117, E00H15. https://doi.org/10.1029/2011JE003916

Hawke, B. R., Blewett, D. T., Lucey, P. G., Smith, G. A., Bell III, J. F., Campbell, B. A., \& Robinson, M. S. (2004). The origin of lunar crater rays. Icarus, 170, 1-16.

Hawkins, S. E., Boldt, J. D., Darlington, E. H., Espiritu, R., Gold, R. E., Gotwols, B., et al. (2007). The Mercury Dual Imaging System on the MESSENGER Spacecraft. Space Science Reviews, 131(1-4), 247-338. https://doi.org/10.1007/s11214-007-9266-3

Head, J. W., Murchie, S. L., Prockter, L. M., Robinson, M. S., Solomon, S. C., Strom, R. G., et al. (2008). Volcanism on Mercury: Evidence from the first MESSENGER flyby. Science, 321, 69-72.

Head, J. W., Murchie, S. L., Prockter, L. M., Solomon, S. C., Chapman, C. R., Strom, R. G., et al. (2009). Volcanism on Mercury: Evidence from the first MESSENGER flyby for extrusive and explosive activity and the volcanic origin of plains. Earth and Planetary Science Letters, 285, 227-242.

Izawa, M. R. M., Craig, M. A., Applin, D. M., Sanchez, J. A., Reddy, V., Le Corre, L., et al. (2015). Variability, absorption features, and parent body searches in "spectrally featureless" meteorite reflectance spectra: Case study - Tagish Lake. Icarus, 254, $324-332$.

Izenberg, N. R., Klima, R. L., Murchie, S. L., Blewett, D. T., Holsclaw, G. M., McClintock, W. E., et al. (2014). The low-iron, reduced surface of Mercury as seen in spectral reflectance by MESSENGER. Icarus, 228, 364-374. https://doi.org/10.1016/j.icarus.2013.10.023

Kaasalainen, M., Torppa, J., \& Muinonen, K. (2001). Optimization methods for asteroid lightcurve inversion. Icarus, 153 , 37-51.

Kerber, L., Head, J. W., Blewett, D. T., Solomon, S. C., Wilson, L., Murchie, S. L., et al. (2011). The global distribution of pyroclastic deposits on Mercury: The view from MESSENGER flybys 1-3. Planetary and Space Science, 59(15), 1895-1909. https://doi.org/10.1016/j.pss.2011. 03.020

Kerber, L., Head, J. W., Solomon, S. C., Murchie, S. L., Blewett, D. T., \& Wilson, L. (2009). Explosive volcanic eruptions on Mercury: Eruption conditions, magma volatile content, and implications for interior volatile abundances. Earth and Planetary Science Letters, $285,263-271$.

Klima, R. L., Denevi, B. W., Ernst, C. M., Murchie, S. L., \& Peplowski, P. N. (2018). Global distribution and spectral properties of low-reflectance material on Mercury. Geophysical Research Letters, 45, 2945-2953. https://doi.org/10.1002/2018GL077544

Lantz, C., Brunetto, R., Barucci, M. A., Dartois, E., Duprat, J., Engrand, C., et al. (2015). Ion irradiation of the Murchison meteorite: Visible to mid-infrared spectroscopic results. Astronomy \& Astrophysics, 577, 9.

Lucchetti, A., Pajola, M., Galluzzi, V., Giacomini, L., Carli, C., Cremonese, G., et al. (2018). Mercury hollows as remnants of original bedrock materials and devolatilization processes: A spectral clustering and geomorphological analysis. Journal of Geophysical Research: Planets, 123, 2365-2379. https://doi.org/10.1029/2018je005722

Lucey, P. G., Taylor, GJ., \& Malaret, E. (1995). Abundance and distribution of iron on the Moon. Science, 268, $1150-1154$.

Marzo, G. A., Roush, T. L., Blanco, A., Fonti, S., \& Orofino, V. (2006). Cluster analysis of planetary remote sensing spectral data. Journal of Geophysical Research E, 111, 1-14. https://doi.org/10.1029/2005JE002532

Marzo, G. A., Roush, T. L., Blanco, A., Fonti, S., \& Orofino, V. (2008). Statistical exploration and volume reduction of planetary remote sensing spectral data. Journal of Geophysical Research E, 113, E12009. https://doi.org/10.1029/2008JE003219

Marzo, G. A., Roush, T. L., \& Hogan, R. C. (2009). Automated classification of visible and infrared spectra using cluster analysis. Journal of Geophysical Research, 114, E08001. https://doi.org/10.1029/2008JE003250

McCauley, J. F., Guest, J. E., Schaber, G. G., \& Trask, N. J. (1981). Stratigraphy of the Caloris Basin, Mercury. Icarus, 47, 184-202.

McClintock, W. E., Izenberg, N. R., Holsclaw, G. M., Blewett, D. T., Domingue, D. L., Head, J. W. III, et al. (2008). Spectroscopic observations of Mercury's surface reflectance during MESSENGER's first Mercury flyby. Science, 321, 62-65.

Moroz, L., Baratta, G., Strazzulla, G., Starukhina, L., Dotto, E., Barucci, M. A., et al. (2004). Optical alteration of complex organics induced by ion irradiation: 1. Laboratory experiments suggest unusual space weathering trend. Icarus, 170, 214-228.

Murchie, S. L., Klima, R. L., Denevi, B. W., Ernst, C. M., Keller, M. R., Domingue, D. L., et al. (2015). Orbital multispectral mapping of Mercury with the MESSENGER Mercury Dual Imaging System: Evidence for the origins of plains units and low-reflectance material. Icarus, 254, 287-305. https://doi.org/10.1016/j.icarus.2015.03.027

Nittler, L. R., Starr, R. D., Weider, S. Z., McCoy, T. J., Boynton, W. V., Ebel, D. S., et al. (2011). The major-element composition of Mercury's surface from MESSENGER X-ray spectrometry. Science, 333(6051), 1847-1850. https://doi.org/10.1126/science.1211567

Nittler, L. R., Weider, S. Z., Starr, R. D., Chabot, N., Denevi, B. W., Ernst, C. M., et al. (2014). Sulfur-depleted composition of Mercury's largest pyroclastic deposit: Implications for explosive volcanism and surface reflectance on the innermost planet, Lunar and Planetary Science XLV, abstract \#1391.

Peplowski, P. N., Blewett, D. T., Denevi, B. W., Evans, L. G., Lawrence, D. J., Nittler, L. R., et al. (2011). Mapping iron abundances on the surface of Mercury: Predicted spatial resolution of the MESSENGER Gamma-Ray Spectrometer. Planetary and Space Science, 59(13), 1654-1658. https://doi.org/10.1016/j.pss.2011.06.001

Peplowski, P. N., Klima, R. L., Lawrence, D. J., Ernst, C. M., Denevi, B. W., Frank, E. A., et al. (2016). Remote sensing evidence for an ancient carbon-bearing crust on Mercury. Nature Geoscience, 9(4), 273-276. https://doi.org/10.1038/ngeo2669

Peplowski, P. N., \& Stockstill-Cahill, K. R. (2018). Mercury's geochemical terranes revisited, Mercury: Current and Future Science of the Innermost Planet, abstract \#6032.

Pieters, C. M., \& Noble, S. K. (2016). Space weathering on airless bodies. Journal of Geophysical Research: Planets, 121, 1865-1884. https:// doi.org/10.1002/2016JE005128 
Pieters, C. M., Staid, M. I., Fischer, E. M., Tompkins, S., \& He, G. (1994). A sharper view of impact craters from Clementine data. Science, 266(5192), 1844-1848. https://doi.org/10.1126/science.266.5192.1844

Potter, W. K., \& Head, J. W. (2017). Basin formation on Mercury: Caloris and the origin of its low-reflectance material. Earth and Planetary Science Letters, 474, 427-435.

Preusker, F., Oberst, J., Stark, A., Matz, K-D., Gwinner, K., \& Roatsch, T. (2017). High-resolution topography from MESSENGER orbital stereo imaging-The H3 quadrangle "Shakespeare", Lunar and Planetary Science XLVIII, abstract \#1441.

Robinson, M. S., Murchie, S. L., Blewett, D. T., Domingue, D. L., Hawkins, S. Edward, Head, J. W., et al. (2008). Reflectance and color variations on Mercury: Regolith processes and compositional heterogeneity. Science, 321(5885), 66-69. https://doi.org/10.1126/science. 1160080

Shkuratov, Y. G., \& Helfenstein, P. (2001). The opposition effect and the quasi-fractal structure of regolith: I. Theory. Icarus, $152,96-116$.

Solomon, S. C., McNutt, RalphL. Jr., Gold, R. E., \& Domingue, D. L. (2007). MESSENGER mission overview. Space Science Reviews, 131, 3-39. https://doi.org/10.1007/s11214-007-9247-6

Spudis, P. D., \& Guest, J. E. (1988). Stratigraphy and geological history of Mercury. In F. Vilas, C. R. Chapman, \& M. S. Matthews (Eds.), Mercury (pp. 118-164). Tucson: University of Arizona Press.

Thomas, R., Hynek, B. M., Rothery, D. A., \& Conway, S. J. (2016). Mercury's low-reflectance material: Constraints from hollows. Icarus, 277, 455-465. https://doi.org/10.1016/j.icarus.2016.05.036

Thomas, R. J., Rothery, D. A., Conway, S. J., \& Anand, M. (2014). Hollows on Mercury: Materials and mechanisms involved in their formation. Icarus, 229, 221-235. https://doi.org/10.1016/j.icarus.2013.11.018

Torson, J. M., \& Becker, K. J. (1997). ISIS-A software architecture for processing planetary images, Lunar and Planetary Science XXVIII, abstract \#1219.

Tou, J. T., \& Gonzalez, R. C. (1974). Pattern recognition principles. Applied Mathematics and Computation, 7, 395.

Trang, D., Lucey, P. G., \& Izenberg, N. R. (2017). Radiative transfer modeling of MESSENGER VIRS spectra: Detection and mapping of submicroscopic iron and carbon. Icarus, 293, 206-217.

Trask, N. J., \& Guest, J. E. (1975). Preliminary geologic terrain map of Mercury. Journal of Geophysical Research, 80(17), 2461-2477. https:// doi.org/10.1029/JB080i017p02461

Vander Kaaden, K. E., \& McCubbin, F. M. (2015). Exotic crust formation on Mercury: Consequences of a shallow, FeO-poor mantle. Journal of Geophysical Research: Planets, 120, 1-15. https://doi.org/10.1002/2014JE004733

Vilas, F., Domingue, D. L., Helbert, J., D'Amore, M., Maturilli, A., Klima, R. L., et al. (2016). Mineralogical indicators of Mercury's hollows composition in MESSENGER color observations. Geophysical Research Letters, 43, 1450-1456. https://doi.org/10.1002/2015GL067515

Weider, S. Z., Nittler, L. R., Starr, R. D., Crapster-Pregont, E. J., Peplowski, P. N., Denevi, B. W., et al. (2015). Evidence for geochemical terranes on Mercury: Global mapping of major elements with MESSENGER's X-Ray Spectrometer. Earth and Planetary Science Letters, 416, 109-120. https://doi.org/10.1016/j.epsl.2015.01.023

Weider, S. Z., Nittler, L. R., Starr, R. D., McCoy, T. J., \& Solomon, S. C. (2014). Variations in the abundance of iron on Mercury's surface from MESSENGER X-Ray Spectrometer observations. Icarus, 235, 170-186. https://doi.org/10.1016/j.icarus.2014.03.002

Weider, S. Z., Nittler, L. R., Starr, R. D., McCoy, T. J., Stockstill-Cahill, K. R., Byrne, P. K., et al. (2012). Chemical heterogeneity on Mercury's surface revealed by the MESSENGER X-Ray Spectrometer. Journal of Geophysical Research E, 117, E00L05. https://doi.org/10.1029/ 2012JE004153

Xiao, Z., Prieur, N. C., \& Werner, S. C. (2016). The self-secondary crater population of the Hokusai crater on Mercury. Geophysical Research Letters, 43, 7424-7432. https://doi.org/10.1002/2016GL069868

Xiao, Z., Strom, R. G., Blewett, D. T., Byrne, P. K., Solomon, S. C., Murchie, S. L., et al. (2013). Dark spots on Mercury: A distinctive low-reflectance material and its relation to hollows. Journal of Geophysical Research: Planets, 118, 1752-1765. https://doi.org/10.1002/ jgre.20115

Xiao, Z., Zeng, Z., Li, Z., Blair, D. M., Xiao, L., Zeng, Z., et al. (2014). Cooling fractures in impact melt deposits on the Moon and Mercury: Implications for cooling solely by thermal radiation. Journal of Geophysical Research: Planets, 119, 1496-1515. https://doi.org/10.1002/ 2013JE004560

Zambon, F., Galluzzi, V., Carli, C., Capaccioni, F., Wright, J., \& Rothery, D. A. (2017). Spectral variations on H-2 Victoria quadrangle on Mercury: The case of Hokusai rays. European Planetary Science Congress 2017 (Riga), EPSC2017-903-3. 